SAND98-1189

Printed June 1998

$$
\text { SAND }-98-1189
$$

\title{
A Survey of Probabilistic Methods Used in Reliability, Risk and Uncertainty Analysis: Analytical Techniques I
}

David G. Robinson

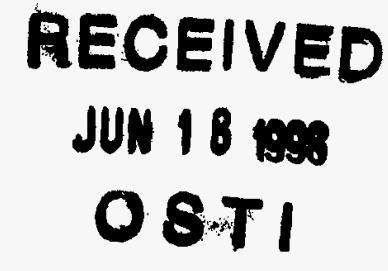

Prepared by

Sandia National Laboratories

Albuquerque, New Mexico 87185 and Livermore, California 94550

Sandia is a multiprogram laboratory operated by Sandia Corporation, a Lockheed Martin Company, for the United States Department of

Energy under Contract DE-AC04-94AL85000.

Approved for public release; further dissemination unlimited.

\section{Sandia National Laboratories}

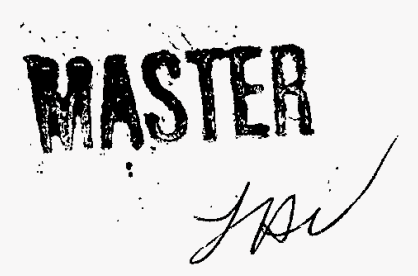


Issued by Sandia National Laboratories, operated for the United States Department of Energy by Sandia Corporation.

NOTICE: This report was prepared as an account of work sponsored by an agency of the United States Government. Neither the United States Government nor any agency thereof, nor any of their employees, nor any of their contractors, subcontractors, or their employees, makes any warranty, express or implied, or assumes any legal liability or responsibility for the accuracy, completeness, or usefulness of any information, apparatus, product, or process disclosed, or represents that its use would not infringe privately owned rights. Reference herein to any specific commercial product, process, or service by trade name, trademark, manufacturer, or otherwise, does not necessarily constitute or imply its endorsement, recommendation, or favoring by the United States Government, any agency thereof, or any of their contractors or subcontractors. The views and opinions expressed herein do not necessarily state or reflect those of the United States Government, any agency thereof, or any of their contractors.

Printed in the United States of America. This report has been reproduced directly from the best available copy.

Available to DOE and DOE contractors from

Office of Scientific and Technical Information

P.O. Box 62

Oak Ridge, TN 37831

Prices available from (615) 576-8401, FTS 626-8401

Available to the public from

National Technical Information Service

U.S. Department of Commerce

5285 Port Royal Rd

Springfield, VA 22161

NTIS price codes

Printed copy: A03

Microfiche copy: A01

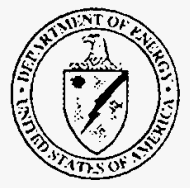


SAND98-1189

Unlimited Release

Printed June 1998

\title{
A Survey of Probabilistic Methods Used In Reliability, Risk and Uncertainty Analysis: Analytical Techniques I
}

\author{
David G. Robinson \\ Systems Reliability \\ Sandia National Laboratories \\ P.O. Box 5800 \\ Albuquerque, NM 87185-0746 \\ drobin@sandia.gov
}

\begin{abstract}
This report provides an introduction to the various probabilistic methods developed roughly between 1956-1985 for performing reliability or probabilistic uncertainty analysis on complex systems. This exposition does not include the traditional reliability methods (e.g. parallel-series systems, etc.) that might be found in the many reliability texts and reference materials (e.g. Kapur and Lamberson, 1977). Rather, the report centers on the relatively new, and certainly less well known across the engineering community, analytical techniques. Discussion of the analytical methods has been broken into two reports. This particular report is limited to those methods developed between 1956-1985. While a bit dated, methods described in the later portions of this report still dominate the literature and provide a necessary technical foundation for more current research. A second report (Analytical Techniques II) addresses methods developed since 1985. The flow of this report roughly follows the historical development of the various methods so each new technique builds on the discussion of strengths and weaknesses of previous techniques. To facilitate the understanding of the various methods discussed, a simple 2-dimensional problem is used throughout the report. The problem is used for discussion purposes only; conclusions regarding the applicability and efficiency of particular methods are based on secondary analyses and a number of years of experience by the author.

This document should be considered a 'living document' in the sense that as new methods or variations of existing methods are developed, the document and references will be updated to reflect the current state of the literature as much as possible. For those scientists and engineers already familiar with these methods, the discussion will at times become rather obvious. However, the goal of this effort is to provide a common basis for future discussions and, as such, will hopefully be useful to those more intimate with probabilistic analysis and design techniques. There are clearly alternative methods of dealing with uncertainty (e.g. fuzzy set theory, possibility theory), but this discussion will be limited to those methods based on probability theory.
\end{abstract}




\section{Contents}

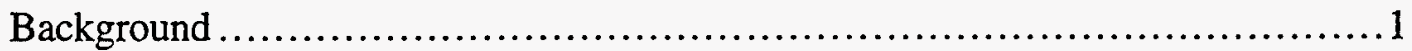

Types of Uncertainty ................................................

Sources of Uncertainty ..................................................

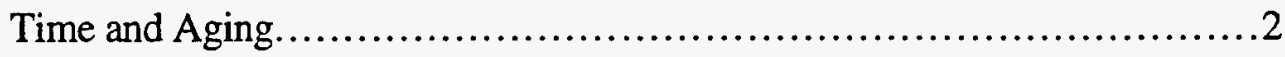

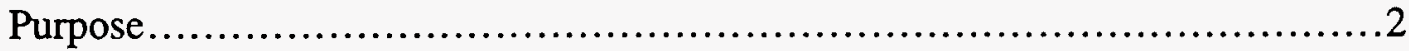

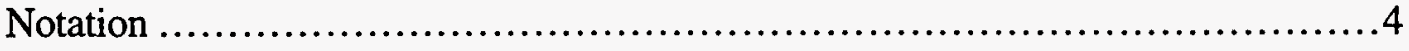

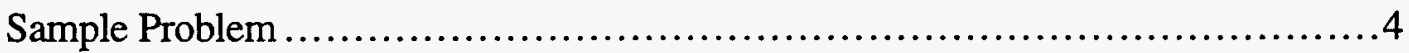

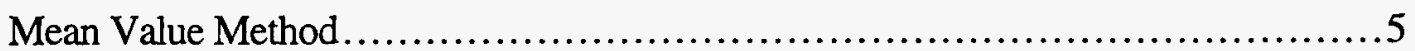

Development ..........................................................

Application ....................................................... 6

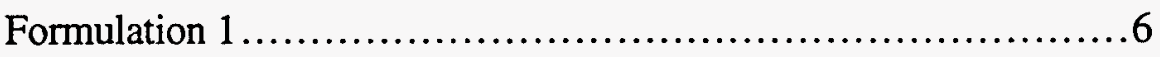

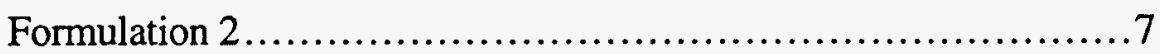

Response Surface Methods ............................................... 8

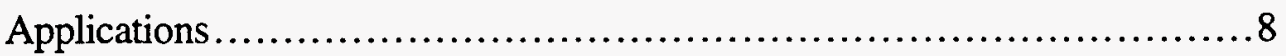

Construction of the Experimental Matrix ............................. 10

Differential Analysis ........................................................ 12

First-Order Reliability Methods............................................... 12

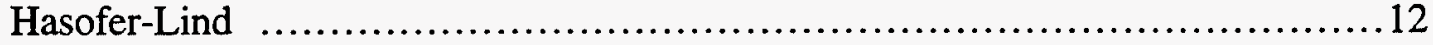

Application ............................................................. 17

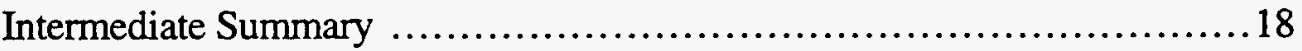

Sensitivity Analysis ................................................ 18

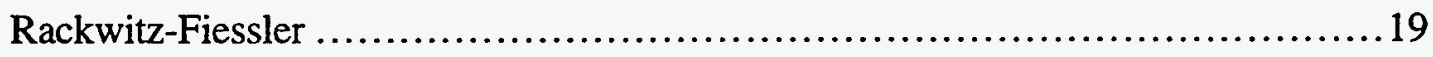

Advanced First Order Reliability Method.....................................20

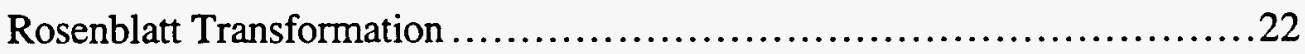

Rosenblatt Transformation Examples.............................23

AFORM Algorithm................................................. 24

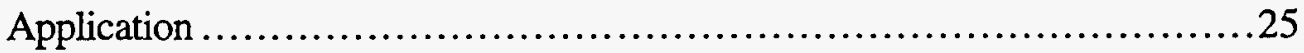

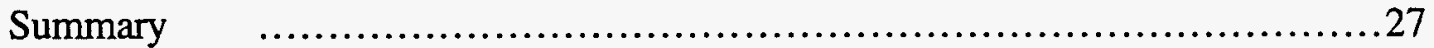

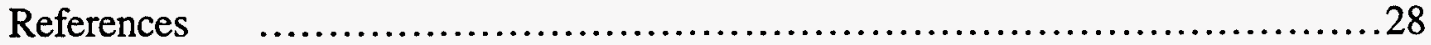




\section{DISCLAIMER}

This report was prepared as an account of work sponsored by an agency of the United States Government. Neither the United States Government nor any agency thereof, nor any of their employees, makes any warranty, express or implied, or assumes any legal liability or responsibility for the accuracy, completeness, or usefulness of any information, apparatus, product, or process disclosed, or represents that its use would not infringe privately owned rights. Reference herein to any specific commercial product, process, or service by trade name, trademark, manufacturer, or otherwise does not necessarily constitute or imply its endorsement, recommendation, or favoring by the United States Government or any agency thereof. The views and opinions of authors expressed herein do not necessarily state or reflect those of the United States Government or any agency thereof. 


\section{Figures}

Figure 1. $\mathrm{G}(\mathrm{x})$ for Sample Problem..........................................

Figure 2. RS Approximation vs. Original Performance Function....................9

Figure 3. 3D View of RS Model ................................................. 10

Figure 4. Limit State Function................................................ 13

Figure 5. New Limit State Function .......................................... 13

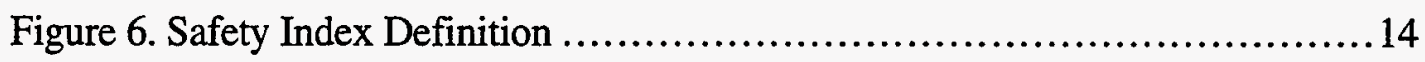

Figure 7. Depiction of Joint Probability Density Function .......................... 14

Figure 8. New Limit State Function and Distance Function ........................ 18

Figure 9. Depiction of Sensitivity Coefficients .....................................19

Figure 10. Gaussian Approximation............................................ 20

Figure 11. Equivalent Normal Approximation..................................20

\section{Tables}

Table 1. H-L Application Results ............................................... 17

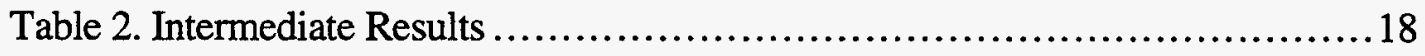

Table 3. Results of Rackwitz-Fiessler Method .................................22

Table 4. Summary of Hohenbichler-Rackwitz Iterations...........................27 


\section{A Survey of Probabilistic Methods Used In Reliability, Risk and Uncertainty Analysis: Analytical Techniques I}

\section{Background}

\section{Types of Uncertainty}

Uncertainties typically fall into one of two categories: probabilistic or possibilistic. However, even some system aspects that are clearly deterministic, such as the use of a pure sine wave to model system input, can be characterized using probabilistic methods. Probabilistic techniques are characterized by the use of random variables to describe the various sources of uncertainty and are often referred to as reliability methods_by structural engineers. These techniques are typically applied when the system under consideration is of small to moderate complexity $(100-150$ random variables) and is reasonably well understood. Possibilistic techniques involve the use of fuzzy set theory or possibility theory to model uncertainty and are particularly useful when dealing with large, complex systems. In most cases, an exact system model may be impractical or even impossible to obtain.

\section{Sources of Uncertainty}

Discounting ignorance on the part of the user, uncertainty arises from four major sources. External system parameters are very common sources of uncertainty and include a variety of possibilities: temperature, radiation, road surface, etc. While the engineer can select certain materials for their strength, wear or corrosion characteristics, the true characteristics can vary considerably when the system is finally constructed. Material properties are only one example of uncertainty in internal system parameters.

External and internal sources of uncertainty can be roughly characterized by the level of control the designer has over the range of values that the system might experience. For example, temperature might be external or internal depending on the ability of the system designer to regulate the extreme temperatures that the system might be exposed to. In a sense, the more control the engineer has over the external variables, the more they become part of, or internal to, the system being designed

A third source of uncertainty is the system model. By definition the model is only an abstract representation of the real world. The choice of modeling technique or the level of detail used within a model might lead to error in the estimated response of the system. For example, even finite element models with hundreds of thousands of elements provide a very simple representation of how the physical system will behave. While appearing to be deterministic, such modeling uncertainty can often be characterized using various probabilistic or possibilistic analysis techniques.

A final source of uncertainty is introduced when the engineer attempts to observe the behavior of the system. Such things as human perception, measurement error, or sensor error compounded by data fusion are significant sources of observational uncertainty. 


\section{Analytical Techniques I}

\section{Time and Aging}

Characterization of the uncertainties inherent in the system and its operating environment is complicated by time. As the system ages or goes through various upgrades and redesigns, the uncertainties will also continuously change.

During the initial stages of design, the system progresses through a test-redesign-test cycle. As testing progresses, faults and design weaknesses are found and design changes are made. Testing begins again and the cycle continues until the system demonstrates the required reliability characteristics or it is impractical (i.e. not cost effective) to continue redesign. This process is referred to as reliability growth. However, as soon as the system become operational the system begins to age. As time passes the microstructure will evolve, corrosion will invade connections and surfaces will wear. It becomes a constant battle to maintain and support the system; typically the majority of the costs of a system are incurred during this stage of the system life cycle.

Attention during the design of a system to the inevitable problems associated with aging and wearout can alleviate much of this cost. In addition, by proper application of uncertainty techniques later in the system life cycle, failures can be anticipated and addressed pro-actively; generally at significant savings relative to the costs incurred as a result of a catastrophic system failure.

However, a detailed discussion of the implications of time dependent system uncertainties is beyond the scope of this current effort and is left to a follow-on report.

\section{Purpose}

The purpose of this current report is to document the various methods available for performing reliability analysis or probabilistic uncertainty analysis on complex systems. This exposition does not include the traditional reliability methods that might be found in the many reliability texts and reference materials (e.g. Kapur and Lamberson, 1977). Rather, the following discussion centers on relatively new, and certainly less well known across the engineering community, reliability analysis techniques. The discussion is directed at the engineer or analyst whose background may involve an undergraduate probability course. The report may therefore appear to be simplistic to those with a much stronger probability and statistics background.

This document should be considered a 'living document' in the sense that as new methods or variations of existing methods are developed, the document and references will be updated to reflect the current state of the literature as much as possible. For those scientists and engineers already familiar with these methods, the discussion will at times become rather obvious.

However, the goal of this effort is to provide a common basis for future discussions and, as such, will hopefully be useful to those more intimate with probabilistic analysis and design techniques. There are clearly alternative methods of dealing with uncertainty (e.g. fuzzy set theory, possibility theory), but this discussion will be limited to those methods based on probability theory.

The primary goal of all of these methods is the characterization of uncertainty in system response due to uncertainty in either internal system parameters (e.g. grain size, yield strength), or external parameters (e.g. temperature, load). These two sources of uncertainty will be grouped into a general vector of uncertainty variables, $\mathbf{x}=\left(x_{1}, x_{2}, \ldots, x_{n}\right)$. The variations in these variables may or may not be time dependent. The response of the system to a particular realization of this vector will be denoted $G(\mathbf{x})$. The initial discussion will concentrate on those situations where the uncertainties can be described independent of time; the discussion will be expanded in a sequel to include time dependent system behavior.

A second goal, equally important during system design, is the sensitivity analysis of system response to uncertainties in the system variables.

Probabilistic methods are typically applied to address one of two basic questions: What does the probability density function of the system response look like? Or, alternatively, and at first glance a 
bit more narrow in scope: What is the probability that the system response exceeds a critical level? From a reliability engineering design and analysis point of view, the latter approach is the most common since reliability engineers are typically responsible for describing the probability of system failure relative to a set of design codes or specifications. Interest in system response is typically focused on a particular region of interest rather than the entire domain of possible system responses. Further, by examining a number of possible 'critical levels', clearly the density function of the system response can be completely characterized (rather than evaluated at simply one point). For these reasons, the second approach will be emphasized in this discussion. When a particular technique provides additional benefits beyond this objective, these will be highlighted as much as possible.

Assuming the existence (real or artificial) of a critical level of system performance, the result is the partitioning of the system parameter domain $\mathrm{x}=\left(x_{1}, x_{2}, \ldots, x_{n}\right)$ into two regions: a region $\Omega$ where combinations of system parameters lead to an unacceptable or unsafe system response and a safe region $\bar{\Omega}$ where system response is acceptable. The surface dividing these regions is generally referred to as the limit state surface or limit state function. The probability of system failure is then defined by the expression:

$$
p_{f}=\iint \cdots \int_{\Omega} f_{\mathbf{X}}(\mathbf{x}) d \mathbf{x}
$$

where $f_{\mathbf{X}}(\mathbf{x})$ is the joint probability density function (pdf). Except for some unique limit state functions and joint density functions (e.g. linear limit state and Gaussian distributed random variables), the integral can be very difficult to evaluate. The following discussion centers on the various methods often used to evaluate this integral.

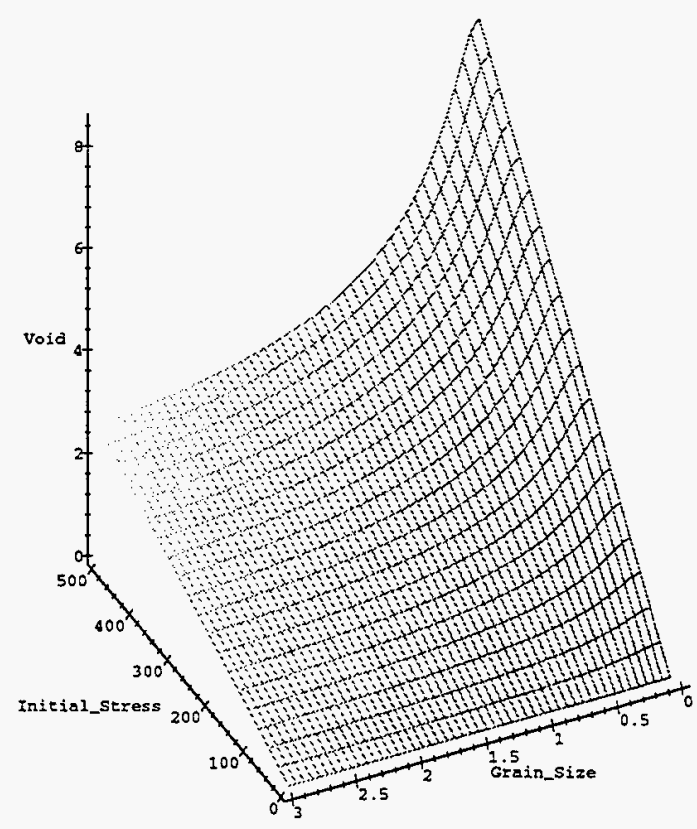

Figure 1. $\mathbf{G}(\mathbf{X})$ for Sample Problem
There are two major categories of probabilistic methods. The first category includes a broad class of random sampling methods such as classic Monte Carlo or importance sampling techniques. These are characterized by the random selection of observations of each system parameter $x_{i}$ such that $f_{\mathbf{X}}(\mathbf{x})$ is satisfied, and these values are subsequently operated on by the system performance function $G(\mathbf{x})$.

The second category of probabilistic methods is characterized by the use of analytical techniques to find a particular point in design space that can be related (at least approximately) to the probability of system failure. This point is often referred to as the most probable point or the design point.

Both of these analysis categories will be discussed in enough detail to permit the reader to apply the techniques to simple problems. The discussion will borrow heavily from the available literature, but a concerted effort will be made to standardize the notation and terminology. Significant differences will be noted where appropriate. For purposes of discussion and to ease the 
introduction of the techniques to the uninitiated, a single sample problem will be used throughout the discussion. Variations on this problem may be introduced to illustrate a particular aspect of a solution methodology.

\section{Notation}

The notation used in the following discussion is consistent with the literature; however, it can be confusing to the uninitiated. The following definitions will be consistent throughout.

$\mathbf{x}=\left\{x_{1}, \ldots, x_{n}\right\}:$ a vector of independent random variables generally referred to individually as design variables. The performance of the system is defined in terms of these variables and the locus of all feasible combinations of these variables is referred to as the design space.

$\mathbf{y}=\left\{y_{1}, \ldots, y_{n}\right\}$ : identical to the vector $\mathbf{x}$ with the exception that these random variables are assumed to be statistically dependent.

$\mathbf{u}=\left\{u_{1}, \ldots, u_{n}\right\}$ : a vector of statistically independent random variables with zero mean and unit standard deviation.

$G(\mathbf{x}), g(\mathbf{x}):$ the performance of the system is assumed to be a function of a set of design variables and is referred to as the system performance function or system response function. This function may be explicit or implicit.

$\phi(\cdot)$ : standard normal density function -

$$
\phi(z)=\frac{1}{\sqrt{2 \pi}} \exp \left(-\frac{z^{2}}{2}\right)
$$

$\Phi(\cdot)$ : cumulative normal density function -

$$
\Phi(z)=\frac{1}{\sqrt{2 \pi}} \int_{-\infty}^{z} \exp \left(-\frac{s^{2}}{2}\right) d s
$$

limit state function: a set defined by the locus of points: $G(\mathbf{x})=0$.

failure region: the limit state function divides the feasible space into (at least) two regions: the failure or unsafe region $G(\mathbf{x})<0$ and the safe region: $G(\mathbf{x})>0$. These definitions are based on historical convention rather than a specific requirement. The use of the terms 'failure' is also customary, since only the likelihood of a particular system state may be of interest rather than system failure.

safety index: $\beta$, the safety index, is defined as the scalar distance, in standard normal space, from the origin to the limit state function. When used in the context of $\Phi(-\beta)$ it is assumed that $\beta>0$. For a linear limit state function and Gaussian distributed random variables, it can be shown that $\beta=\frac{\mu_{G}}{\sigma_{G}}$, the ratio of the mean system response and the standard deviation of the system response (both evaluated at the critical or failure point).

\section{Sample Problem}

The problem to be used throughout the discussion involves the growth of voids within an solder interconnect. As a result of the integrated circuit manufacturing process residual stresses between the interconnect and the passivation layer will remain. These residual stresses will then induce voids in the grain interfaces. These voids grow and eventually lead to a loss of transmission capability in the interconnect line. For a specific length of time in storage, line geometry, material properties, the crack size, $\mathrm{A}$, as a function of initial stress, $v_{0}$, and grain size, $L$, is approximated by: 


$$
A=v_{0}\left[0.01694-0.01353 \exp \left(-\frac{0.4158}{L}\right)\right]
$$

Failure of the interconnect then occurs when the crack size exceeds some critical level $A_{c}$ :

$p_{f}=\operatorname{Pr}\left\{A_{c}<A\right\}$ or equivalently:

$p_{f}=\operatorname{Pr}\left\{A_{c}-A<0\right\}=\operatorname{Pr}\left\{A_{c}-v_{0}\left[0.01694-0.01353 \exp \left(-\frac{0.4158}{L}\right)\right]<0\right\}=\operatorname{Pr}\{g(\mathbf{x})<0\}$

(to be consistent with the reliability engineering literature). Note that here $\mathbf{x}=\left\{x_{1}, x_{2}\right\}=\left\{v_{0}, L\right\}$. The integral of interest is therefore:

$$
p_{f}=\iint_{\Omega: G(X)<0} f_{\mathbf{X}}(\mathbf{x}) d \mathbf{x}
$$

where $f_{\mathbf{X}}(\mathbf{x})$ is the joint probability density function of $v_{0}$ and $\mathrm{L}$.

The following discussion is organized into the two broad categories of probabilistic analysis techniques discussed earlier. The analytical methods will be discussed first; following roughly in chronological order of development. As such, each method will develop successively on the weaknesses and strengths of its predecessors.

\section{Mean Value Method}

This method is commonly referred to as the mean value first-order second moment (MVFOSM or simply MV) method since it typically involves a first order expansion about the mean to estimate the first and second moments. It is also closely related to differential analysis techniques (both direct and adjoint). This discussion is based on the material in [Morgan, et al: pp183-192] and [Kapur and Lamberson pp95-121]; there are numerous other sources. In summary the mean value method involves developing the Taylor series expansion of $G(\mathbf{x})$ about the nominal or mean values of the individual random variables. The moments of the resulting approximating function are then easily found. Based on these moments approximate statements can be made regarding the probability of failure. (Note that second order terms are included for completeness.)

\section{Development}

$$
\begin{aligned}
G(\mathbf{x}) & =G\left(x_{1}, \ldots, x_{n}\right) \\
& \left.\approx G(\mathbf{x})\right|_{\mathbf{x}=\bar{\mu}}+\left.\sum_{i=1}^{n} \frac{\partial G(\mathbf{x})}{\partial x_{i}}\right|_{\mathbf{x}=\bar{\mu}}\left(x_{i}-\mu_{i}\right)+\left.\frac{1}{2 !} \sum_{j=1}^{n} \sum_{i=1}^{n} \frac{\partial^{2} G(\mathbf{x})}{\partial x_{i} \partial x_{j}}\right|_{\mathbf{X}=\bar{\mu}}\left(x_{i}-\mu_{i}\right)\left(x_{j}-\mu_{j}\right)+\text { H.O.T. }
\end{aligned}
$$

where: $\bar{\mu}=\left\{\mu_{1}, \ldots, \mu_{n}\right\}$. Taking the expectation of this expression (and neglecting higher order terms):

$$
\begin{aligned}
E[G(\mathbf{x})] & \approx G(\mathbf{x})\left|\mathbf{x}_{\mathbf{x}=\bar{\mu}}+\sum_{i=1}^{n} \frac{\partial G(\mathbf{x})}{\partial x_{i}}\right|_{\mathbf{x}=\bar{\mu}} E\left[\left(x_{i}-\mu_{i}\right)\right]+\left.\frac{1}{2 !} \sum_{j=1}^{n} \sum_{i=1}^{n} \frac{\partial^{2} G(\mathbf{x})}{\partial x_{i} \partial x_{j}}\right|_{\mathbf{x}=\bar{\mu}} E\left[\left(X_{i}-\mu_{i}\right)\left(X_{j}-\mu_{j}\right)\right] \\
& =\left.G(\mathbf{x})\right|_{\mathbf{x}=\bar{\mu}}+\left.\frac{1}{2 !} \sum_{j=1}^{n} \sum_{i=1}^{n} \frac{\partial^{2} G(\mathbf{x})}{\partial x_{i} \partial x_{j}}\right|_{\mathbf{x}=\bar{\mu}} E\left[\left(x_{i}-\mu_{i}\right)\left(x_{j}-\mu_{j}\right)\right]
\end{aligned}
$$


A similar expression for the variance can be found:

$$
\begin{aligned}
V[G(\mathbf{x})] & =E\left[(G(\mathbf{x})-E[G(\mathbf{x})])^{2}\right] \\
& \approx \sum_{j=1}^{n} \sum_{i=1}^{n} \operatorname{Cov}\left[x_{i}, x_{j}\right]\left(\left.\frac{\partial G(\mathbf{x})}{\partial x_{i}}\right|_{\mathbf{x}=\bar{\mu}}\right)\left(\left.\frac{\partial G(\mathbf{x})}{\partial x_{j}}\right|_{\mathbf{x}=\bar{\mu}}\right) \\
& =\sum_{i=1}^{n} V\left[x_{i}\right]\left(\left.\frac{\partial G(\mathbf{x})}{\partial x_{i}}\right|_{\mathbf{x}=\bar{\mu}}\right)^{2}+2 \sum_{j=1}^{n} \sum_{i=j+1}^{n} \operatorname{Cov}\left[x_{i}, x_{j}\right]\left(\left.\frac{\partial G(\mathbf{x})}{\partial x_{i}}\right|_{\mathbf{x}=\bar{\mu}}\right)\left(\left.\frac{\partial G(\mathbf{x})}{\partial x_{j}}\right|_{\mathbf{x}=\bar{\mu}}\right)
\end{aligned}
$$

If the random variables are independent, these expressions can be substantially simplified:

$$
\begin{aligned}
& \left.E[G(\mathbf{x})] \approx G(\mathbf{x})\right|_{\mathbf{x}=\bar{\mu}} \\
& V[G(\mathbf{x})] \approx \sum_{i=1}^{n} V\left[x_{i}\right]\left(\left.\frac{\partial G(\mathbf{x})}{\partial x_{i}}\right|_{\mathbf{x}=\bar{\mu}}\right)^{2}
\end{aligned}
$$

\section{Application}

\section{Formulation 1}

For the discussion here it will suffice to assume that the random variables, $v_{0}$ and $\mathrm{L}$ are independent with known first and second moments, but the exact form of the probability density functions are unknown. The mean and coefficient of variation $(\sigma / \mu)$ for each of the random variables are $-v_{0}: U(300,0.2), L:(1.25,0.1)$. The line width is assumed to be 3 microns and the critical crack length is assumed to occur when it extends across $90 \%$ of the interconnect: $A_{c}=2.7$.

$$
\begin{gathered}
G\left(v_{0}, L\right)=A_{c}-v_{0}\left[0.01694-0.01353 \exp \left(-\frac{0.4158}{L}\right)\right] \\
E\left[G\left(v_{0}, L\right)\right]=\left.\mu_{G} \approx G(\mathbf{x})\right|_{\mathbf{x}=\bar{\mu}} \\
=2.7-\mu_{v_{0}}\left[0.01694-0.01353 \exp \left(-\frac{0.4158}{\mu_{L}}\right)\right] \\
=2.7-300\left[0.01694-0.01353 \exp \left(-\frac{0.4158}{1.25}\right)\right] \\
=0.528417780 \\
V[G(\mathbf{x})]=\sigma_{G}^{2} \approx \sum_{i=1}^{n} V\left[x_{i}\right]\left(\left.\frac{\partial G(\mathbf{x})}{\partial x_{i}}\right|_{x=\bar{\mu}}\right)^{2}=\sigma_{v_{0}}^{2}\left[0.1694+0.01353 \exp \left(-\frac{0.4158}{\mu_{L}}\right)\right]+\sigma_{L}^{2}\left[\mu_{v_{0}} 0.005625774 \exp \left(-\frac{0.4158}{\mu_{L}}\right) / \mu_{L}^{2}\right] \\
=0.1980033634
\end{gathered}
$$


Define the safety index: $\beta=\frac{\mu_{G}}{\sigma_{G}}$. Given the above moments and assuming that the system response, $G(\mathbf{x})$, can be modeled as a normally distributed random variable, approximate statements can then be made regarding the probability of failure:

$$
\begin{aligned}
p_{f} & \approx \operatorname{Pr}\{G(\mathbf{x})<0\}=\operatorname{Pr}\left\{\frac{G(\mathbf{x})-\mu_{G}}{\sigma_{G}}<\frac{0-\mu_{G}}{\sigma_{G}}\right\} \\
& =\Phi\left(-\frac{\mu_{G}}{\sigma_{G}}\right)=\Phi(-\beta)
\end{aligned}
$$

where $\Phi(\cdot)$ is the standard normal cumulative density function. In the example above, the probability of failure is then approximately: $\Phi(-1.1875)=0.11751$. It should be noted that the assumption of normality of the system response, coupled with the use of the Taylor series expansion as an approximation of the system response function, are the major sources of error associated with the use the MV technique.

\section{Formulation 2}

A necessary condition for any uncertainty analysis technique is that it should be independent of the mathematical formulation of the problem as long as the original definition of failure is maintained. That is, the results of analysis of $p_{f}=\operatorname{Pr}\{R<S\}$ should be equivalent to $p_{f}=\operatorname{Pr}\{R-S<0\}$ or $p_{f}=\operatorname{Pr}\left\{\frac{R}{S}<1\right\}$. For example, an equivalent formulation of the problem is given by:

$$
p_{f}=\operatorname{Pr}\left\{A_{c}-A<0\right\}=\operatorname{Pr}\left\{\frac{A_{c}}{v_{0}}-\left[0.01694-0.01353 \exp \left(-\frac{0.4158}{L}\right)\right]<0\right\}
$$

Based on this equivalent formulation, the mean and variance can once again be found:

$$
\begin{aligned}
E\left[G\left(v_{0}, L\right)\right] & =\left.\mu_{G} \approx G(\mathbf{x})\right|_{\mathbf{x}=\bar{\mu}} \\
& =\frac{A_{c}}{\mu_{v_{0}}}-\left[0.01694-0.01353 \exp \left(-\frac{0.4158}{\mu_{L}}\right)\right] \\
& =0.00176139 \\
V[G(\mathbf{x})]= & \sigma_{G}^{2} \approx \sum_{i=1}^{n} V\left[x_{i}\right]\left(\left.\frac{\partial G(\mathbf{x})}{\partial X_{i}}\right|_{\mathbf{x}=\bar{\mu}}\right)^{2}= \\
= & \sigma_{v_{0}}^{2}\left(\frac{2.7}{\mu_{v_{0}}{ }^{2}}\right)+\sigma_{L}^{2}\left[0.005625774 \exp \left(-\frac{0.4158}{\mu_{L}}\right) / \mu_{L}{ }^{2}\right] \\
= & 0.00258166
\end{aligned}
$$

The safety index in this case is: $\beta=\frac{\mu_{G}}{\sigma_{G}}=0.9631944$. Given the above moments, approximate statements can then again be made regarding the probability of failure: $\Phi(-\beta)=0.167725$. 


\section{Analytical Techniques I}

Clearly, when the MV method is applied on two problems that should give identical results, difficulties in estimating the probability of failure can be encountered. The above reformulation is particularly common when engineers are attempting to deal with dimensionless units. The issue of solution sensitivity to problem formulation becomes even more problematic when dealing with very large, complex representations of system behavior.

\section{Response Surface Methods}

The response surface (RS) method is very similar to the MV method described above and can be thought of as a subset of these methods. While the MV methods deal directly with the performance function, the RS approach involves approximating the original, complicated system performance function with a simpler, more computationally tractable system model. This approximation typically takes the form of a first or second order polynomial:

$$
\begin{aligned}
G(\mathbf{x})=G\left(x_{1}, \ldots, x_{n}\right) \approx & a_{0}+a_{1} x_{1}+\cdots+a_{n} x_{n}+ \\
& a_{n+1} x_{1}^{2}+\cdots+a_{2 n} x_{n}^{2}+a_{2 n+1} x_{1} x_{2}+\cdots
\end{aligned}
$$

Determination of the constants is accomplished through a linear regression about some nominal value, typically the mean. Given this new performance function, the analysis then proceeds in exactly the same manner as the mean value method. It should be noted that not all response methods require a polynomial function as an approximation and the response function is not required to be linear in the parameters. The reader should be cautioned that there are additional analytical methods used in uncertainty analysis that are also referred to as 'response surface methods'. These newer methods will be discussed in the First-Order Reliability Methods section presented later in the document.

\section{Application}

As with the MV approach it will suffice to assume that the random variables, $v_{0}$ and $\mathrm{L}$ are independent with known first and second moments, but the exact form of the probability density functions are unknown. The application as well as the underlying statistics of the random variables remain as described earlier; the response function is repeated here for convenience:

$$
G\left(v_{0}, L\right)=A_{c}-v_{0}\left[0.01694-0.01353 \exp \left(-\frac{0.4158}{L}\right)\right]
$$

By perturbing the two input variables about their respective means, the following input data matrix can be constructed:

$$
\mathbf{X}^{\prime}=\left[\begin{array}{cc}
300 & 1.25 \\
360 & 1.25 \\
240 & 1.25 \\
300 & 1.375 \\
300 & 1.125
\end{array}\right]
$$

where the number of rows $n$ corresponds to the number of observations, or in this case the number of times the performance function is exercised. (NOTE: The particular form for the input matrix has been used by structural reliability engineers since the early 1980's. However, Morris (1991) independently formalized the methodology and a discussion of the formal logic behind the construction of the $\mathbf{X}^{\prime}$ matrix is presented at the end of this section.) Using these values as inputs, the response of the system can be determined: 


$$
\mathbf{z}=\left[\begin{array}{lllll}
0.5284 & 0.0941 & 0.9627 & 0.6178 & 0.4228
\end{array}\right]^{T}
$$

If we had been interested in estimating a second order polynomial the matrix would have been defined:

$$
\mathbf{X}=\left[\begin{array}{cccccc}
1 & x_{11} & x_{12} & x_{11}^{2} & x_{12}^{2} & x_{11} x_{12} \\
1 & x_{21} & x_{22} & x_{21}^{2} & x_{22}^{2} & x_{21} x_{22} \\
\vdots & \vdots & \vdots & \vdots & & \vdots \\
1 & x_{n 1} & x_{n 2} & \cdots & & x_{n 1} x_{n 2}
\end{array}\right]
$$

where $\mathrm{n}$ is the number of sample observations. The minimum number of observations to fit a second order polynomial is six. However, for this example, only the linear effects will be included in the model and only the first two columns are needed. In general: $X=\left[1: X^{\prime}\right]$

From classical linear regression, the normal equations can be solved for the coefficients:

$$
\mathbf{a}=\left(\mathbf{X}^{T} \mathbf{X}\right)^{-1} \mathbf{X}^{T} \mathbf{z}=\left[\begin{array}{lll}
1.7219 & -0.0072 & 0.7798
\end{array}\right]^{T}
$$

The approximate system performance function is therefore:

$$
G(\mathbf{x}) \approx G^{\prime}(\mathbf{x})=1.7219-0.0072 v_{0}+0.7798 L
$$

For information purposes, it is interesting to contrast the predicted system performance based on the RSM approximation and the actual system performance:

$$
\begin{aligned}
\text { Predicted Response } & =\left[\begin{array}{lllll}
0.5251 & 0.0909 & 0.9595 & 0.6224 & 0.4277
\end{array}\right]^{T} \\
\text { Actual Response } & =\left[\begin{array}{lllll}
0.5284 & 0.0941 & 0.9627 & 0.6178 & 0.4228
\end{array}\right]^{T}
\end{aligned}
$$

It appears that a fair approximation of the original function has been found in the neighborhood of the mean using simply the linear components of the regression equation. However, this is not always the

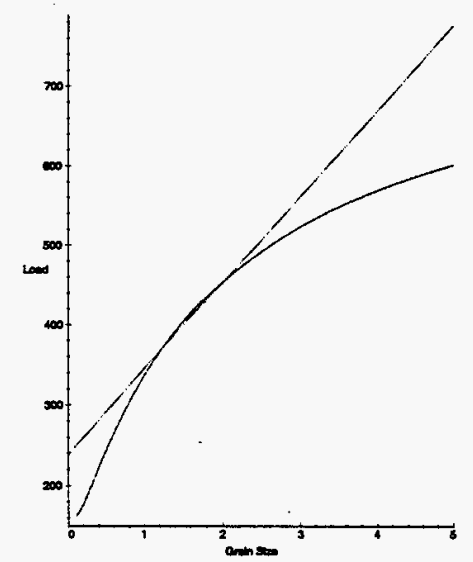

Figure 2. RS Approximation vs. Original Performance Function case given the complex nature of problems encountered in engineering. The level of accuracy is greatly dependent on the magnitude of the perturbation used to construct the input data. The analyst must be aware of the limitations of this approximation and, if possible, a simple validation check is always recommended (e.g. an additional run with a new perturbation or small Monte Carlo sample if economically feasible).

Given an approximation to the original performance function, a number of analysis avenues can be explored. Traditionally, subsequent to finding the regression function, the function can be exercised using Monte Carlo techniques or simply using the MV method previously discussed. The implication of using a vector of mean values as the expansion point is that the system will fail in the neighborhood of the mean. This problem is identical to that experienced when using the MV method. 
There are further directions that can be taken and this will be discussed in detail in a later section. Using the MV technique, the associated moments are easily found (again assuming independence among the random variables):

$$
\begin{aligned}
E\left[G^{\prime}\left(v_{0}, L\right)\right] & =\mu_{G^{\prime}}=\left.G^{\prime}(\mathbf{x})\right|_{\mathbf{x}=\bar{\mu}} \\
& =1.7219-0.0072(300)+0.7798(1.25) \\
& =0.5252 \\
V\left[G^{\prime}(\mathbf{x})\right]= & \sigma_{G^{\prime}}^{2}=\sum_{i=1}^{n} V\left[x_{i}\right]\left(\left.\frac{\partial G^{\prime}(\mathbf{x})}{\partial x_{i}}\right|_{\mathbf{x}=\bar{\mu}}\right)^{2}= \\
= & (60)^{2}(0.0072)^{2}+(0.125)^{2}(0.7798)^{2} \\
= & 0.1981
\end{aligned}
$$

The major benefit of RS type approaches over the MV method is that it is not necessary to have an analytical expression for the performance function to estimate the moments of the system performance function. However, approaches based on this initial regression result will also suffer from the same deficiencies as the MV methods: they are sensitive to the mathematical form used for the performance function. If, for example, the regression equation was based on the second formulation presented in the MV section, the resulting approximation would be quite different. The estimates for the mean and variance would naturally be different also.

Regardless of the problems with formulation conflicts, both the MV and the RS methods suffer from sensitivity to the expansion point. It is clear from Figure 2 (and Figure 3 ) that the approximation osculates with the original function in the area of the mean but has significant error outside the region of the expansion point. Any probabilistic analysis based on expansion about the mean (as is typical in MV or RS techniques) will grossly underestimate the likelihood of failure, unless the underlying performance function is linear and all random variables can be described using the Gaussian density function.

In addition, if the random variables are statistically dependent, the analysis becomes much more difficult. The general approach is to transform the variables into a new space where the variables are approximately independent, perform the MV or RS analysis and then iterate until conversion is reached. It is important to note that the assumption of independence does not guarantee either an optimistic or a conservative estimate of the final failure probability.

\section{Construction of the Experimental Matrix}

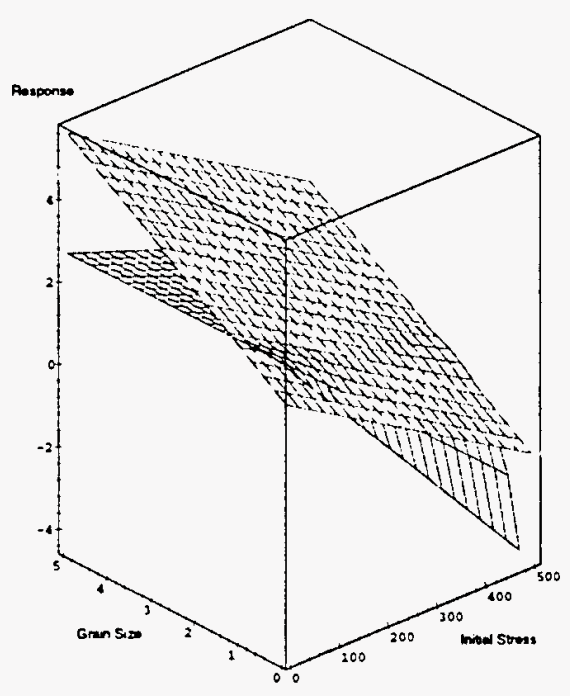

As previously noted, the construction of $\mathbf{X}^{\prime}$ has been fundamental element in structural reliability for a number of years. However, similar efforts have been underway in the area of sensitivity analysis. Morris (1991) suggested an approach for constructing such matrices based on the one-ata-time family of experimental designs. One-at-a-time designs are known to be inefficient when used in linear regression, however, the experiments discussed here involve no random error in the model output so the inefficiencies are not an issue. 
Assume that the number of random variables in the problem is $k$. It is of interest to explore $p$ possible levels or different values of each random variable $i$ across the interval $\left[L_{i}, U_{i}\right]$. The value of $p$ can be different for each random variable, but for our purposes it will be assumed to be both even and constant across all the variables. Define an experiment vector $\mathbf{e}$ composed of integer values from the interval $\left\{0 \leq e_{i} \leq(p-1)\right\}$ each indicating the particular level of $x_{i}$ to apply in the performance function. Define $\Delta$ to be an integer in the interval $\{1,2, \ldots,(p-1)\}$. The goal of the effort is the construction of a $(k+1) \times k$ orientation matrix $\mathbf{B} *$. Each of the rows in $B^{*}$ represent a possible realization of the experimental vector e supporting $(k+1)$ function evaluations.

Define a $(k+1) \times k$ sampling matrix $\mathbf{B}$ as a strictly lower triangular matrix of 1 's:

$\mathbf{B}=\left[\begin{array}{ccccc}0 & 0 & 0 & \cdots & 0 \\ 1 & 0 & 0 & \cdots & 0 \\ 1 & 1 & 0 & \cdots & 0 \\ 1 & 1 & 1 & \cdots & 0 \\ \cdots & \cdots & \cdots & \cdots & \cdots \\ 1 & 1 & 1 & 1 & 1\end{array}\right]$

Let $\mathbf{e}^{*}$ be an initial starting value, which, for the problems considered here is typically a vector associated with the mean values. Further, define $\mathbf{J}_{(k+1), k}$ to be a $(k+1) \times k$ matrix of 1 's and $\mathbf{D} *$ to be a $k$-dimensional diagonal matrix with each element randomly chosen as either -1 or 1 with equal probability. Finally, define $\mathbf{P}$ to be a $k \times k$ matrix obtained by random permuting the columns of a $k \times k$ identity matrix. The orientation matrix is then given by the expression:

$\mathbf{B}^{*}=\left(\mathbf{J}_{(k+1),} \mathbf{l}^{*}+(\Delta / 2)\left[\left(2 \mathbf{B}-\mathbf{J}_{(k+1), k}\right) \mathbf{D}+\mathbf{J}_{(k+1), k}\right]\right) \mathbf{P}$

\section{Example}

Consider a problem involving three random variables. Therefore $k=3$ and let $p=3\left(e_{i} \in\{0,1,2\}\right.$ - low, mean, high) and $\Delta=1$. The $\mathbf{P}$ and $\mathbf{D}$ matrices and the $\mathbf{e}^{*}$ vector are then generated randomly:

$\mathbf{P}=\left[\begin{array}{lll}0 & 1 & 0 \\ 1 & 0 & 0 \\ 0 & 0 & 1\end{array}\right], \quad \mathbf{D}=\left[\begin{array}{ccc}1 & 0 & 0 \\ 0 & -1 & 0 \\ 0 & 0 & 1\end{array}\right], \quad \mathbf{e}^{*}=\left[\begin{array}{lll}1 & 1 & 1\end{array}\right]$.

The resulting orientation matrix is therefore:

$\mathbf{B}^{*}=\left[\begin{array}{lll}2 & 1 & 1 \\ 2 & 2 & 1 \\ 1 & 2 & 1 \\ 2 & 1 & 2\end{array}\right]$.

where each row of $\mathbf{B}^{*}$ corresponds to a particular set of values used to exercise the performance function. These values constitute the experimental matrix, $\mathbf{X}^{\prime}$. 


\section{Differential Analysis}

Differential analysis is not a distinct method for performing uncertainty analysis but is mentioned here since it appears concurrently in a great many uncertainty discussions. It has been used in performing uncertainty analyses for a number of years and, chronologically, became popular at roughly the same time as the MV and RS approaches and is often compared to these methods. A good discussion of the finer points of differential analysis can be found in Zimmerman, et al. (1989), pp14-26. In addition, Iman and Helton $(1985,1988)$ discuss the combination of differential analysis and Monte Carlo methods and compare this combined approach with Latin hypercube sampling.

Differential analysis begins with the application of a Taylor series about some nominal point to approximate the performance function (identical to the MV method). The calculation of the (potentially) numerous partial derivatives is then accomplished using one of two differential analysis techniques: direct or adjoint. Each of these techniques provides some benefits and costs: generally the adjoint method is more computationally efficient. However, as with all methods based on expansion, the primary source of error arises from the choice of expansion point rather than the estimation of the partial derivatives. For application, the reader is referred to the above MV discussion and the SAND report by Zimmerman.

\section{First-Order Reliability Methods}

The following discussions center on a group of analytical techniques loosely referred to as firstorder reliability methods (FORM). These methods are characterized by the iterative, linear approximation to the performance function (hence the term 'first-order'). Fundamentally, these methods can be considered as extensions to the MV methods previously discussed and were developed to address some of the technical difficulties with these methods. The labels associated with each technique are not universal in acceptance and represent what appears to the author to be generally understood phrases used by engineers and analysts when referring to a particular methodology. The methods are presented in roughly a chronological order since each method builds on the previous mathematical developments.

\section{Hasofer-Lind}

One of the major problems with all of the techniques discussed thus far has been the lack of invariance of the solution relative to problem formulation. Simple algebraic changes in the problem formulation can lead to significant changes in assessing the propagation of uncertainty. Hasofer and Lind (1974) presented a methodology which specifically addressed this issue by requiring expansion about a unique point in the feasible solution space. It should be mentioned that Fruedenthal (1956) also proposed a method suggesting similar restrictions on the expansion point.

Fundamental to the approach is the concept of a limit state function. Assuming the existence of a critical level of system performance, the limit state function partitions the system parameter domain $\mathrm{x}=\left(x_{1}, x_{2}, \ldots, x_{n}\right)$ into two regions: a region $\Omega$ where combinations of system parameters lead to an unacceptable or unsafe system response and a safe region $\bar{\Omega}$ where system response is acceptable. The probability of system failure is then defined by the expression: 


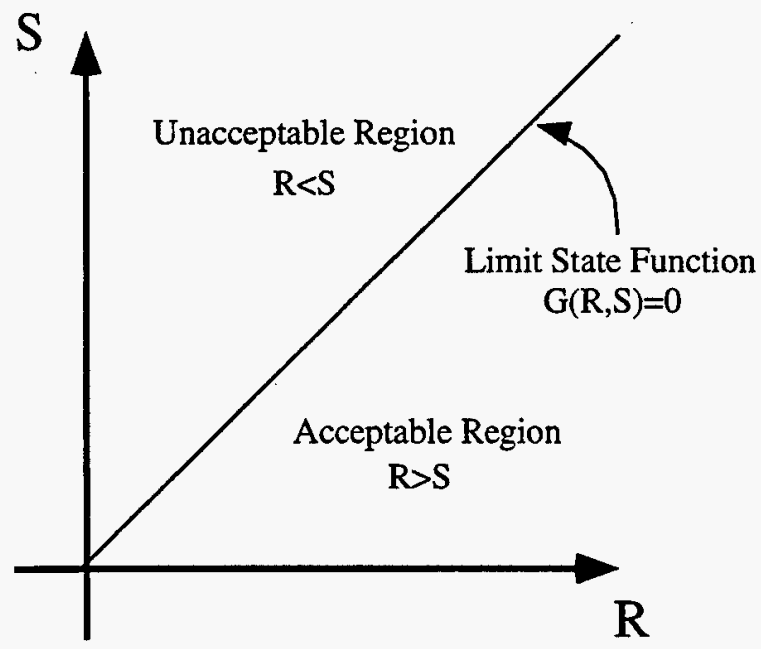

Figure 4. Limit State Function

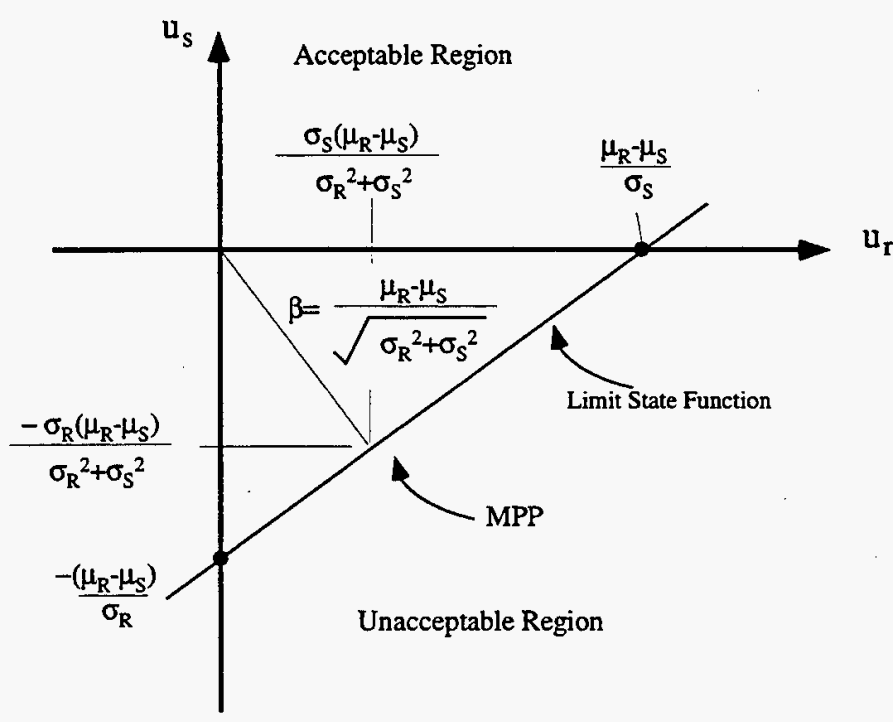

Figure 5. New Limit State Function

$$
p_{f}=\iint \cdots \int_{\Omega} f_{\mathbf{X}}(\mathbf{x}) d \mathbf{x}
$$

Perhaps the simplest way of explaining this approach is to examine the simplest case of two independent random variables. Let $R$ represent a random variable describing the strength of a system and let $S$ represent a random variable describing the stress or load placed on the system. System failure occurs when the stress on the system exceeds the strength of the system: $\Omega=\{(r, s) \mid S>R\}$. Figure 4 depicts the concepts of a limit state function and the associated failure/success regions.

The probability of failure is given by:

$$
\begin{aligned}
p_{f} & =\operatorname{Pr}\{R<S\} \\
& =\operatorname{Pr}\{R-S<0\} \\
& =\operatorname{Pr}\{R / S<1\}
\end{aligned}
$$

where each of the algebraic expressions should give identical results. The limit state function is defined as that locus of points where:

$$
G(R, S): R-S=0
$$

Hasofer and Lind suggested that the first step in overcoming the problem of invariance is to transform the random variables to a set of independent random variables through an orthogonal transformation. One possible transformation, the Rosenblatt transformation, is discussed in a later section. For now, assume that it is possible to transform the system variables to a set of independent random variables. These random variables are then normalized into a set of reduced variables through the transformation: $u_{i}=\frac{x_{i}-\mu_{i}}{\sigma_{i}}$,

where $\mu_{i}$ and $\sigma_{i}$ are respectively the mean and standard deviation of the random variable, $X_{i}$. A new limit state function is then defined in terms of the reduced variables. In the above example, the limit state function becomes the locus of points where:

$$
G\left(\mu_{R}+u_{r} \sigma_{R}, \mu_{s}+u_{s} \sigma_{s}\right)=G(r, s)=\left(\mu_{R}-u_{r} \sigma_{R}\right)-\left(\mu_{s}-u_{s} \sigma_{s}\right)=0
$$

and: $u_{r}=\frac{R-\mu_{R}}{\sigma_{R}}$ and $u_{s}=\frac{S-\mu_{s}}{\sigma_{S}}$. Since this is a linear transformation, no information has been lost. Graphically, the new limit state function appears in Figure 5. In the new space of basic variables, let the minimum distance from the origin to the limit state function be $|\beta|$ (see Figure 6.) 


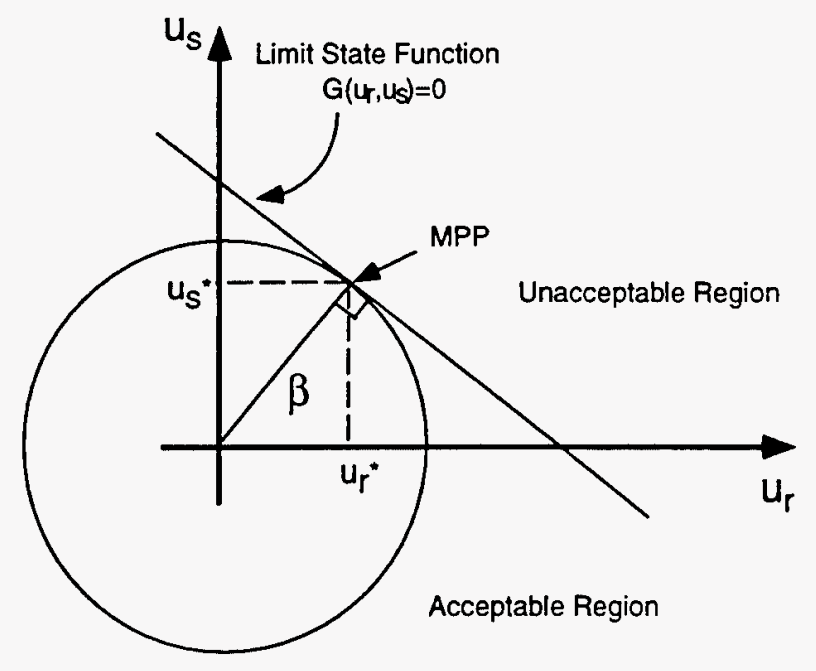

Figure 6. Safety Index

The point on the limit state that lies closest to the origin, $\mathbf{u}^{*}=\left(u_{1}^{*}, u_{2}^{*}, \ldots, u_{n}^{*}\right)$, is often referred to as the most probable point (MPP).

There is a direct relationship between the safety index and the probability of failure. As depicted in Figure 6 (and Figure 7), as $\beta$ increases the limit state moves away from the origin and the probability of failure decreases. In general, this relationship is only approximate, but in the unique case of a linear limit state function and Gaussian distributed random variables, the relationship is exact: $p_{f}=\Phi(-\beta)$. In any case, this situation provides a convenient foundation for developing preliminary skills in analytical uncertainty methods. In addition, many preliminary analyses can be accomplished using these assumptions.

Returning to the simple example with two independent random variables, it is a simple problem in analytical geometry to show that the minimum distance from the origin to the limit state surface in reduced space is:

$$
d=\frac{\mu_{R}-\mu_{S}}{\sqrt{\sigma_{R}^{2}+\sigma_{s}^{2}}}=\frac{\mu_{G}}{\sigma_{G}}=\beta
$$

As previously mentioned, given a linear limit state and independent Gaussian distributed random variables, the probability of failure is given by:

$$
\begin{aligned}
p_{f} & =\int_{\beta}^{\infty} \frac{1}{\sqrt{2 \pi}} \exp \left(-\frac{w^{2}}{2}\right) d w \\
& =\Phi(-\beta)
\end{aligned}
$$

In general, the distance from the point $\mathbf{u}^{*}$ to the limit state $G(\mathbf{x})=0$ is given by the expression:

$$
d=\sqrt{\sum_{i=1}^{n} u_{i}^{*}}
$$

The difficulty then lies in determining the minimum distance for a general nonlinear function. This is essentially a nonlinear, constrained optimization problem:

$$
\text { minimize : } d=\sqrt{\sum_{i=1}^{n} u_{i}^{*}}=\left(\mathbf{u}^{*} \mathbf{u}^{*}\right)^{1 / 2}
$$$$
\text { subject to : } G(\mathbf{u})=0 \quad \text { (equivalently } G(\mathbf{x})=0 \text { ) }
$$

Shinozuka (1983) demonstrated that, in general, the minimum distance is given by:

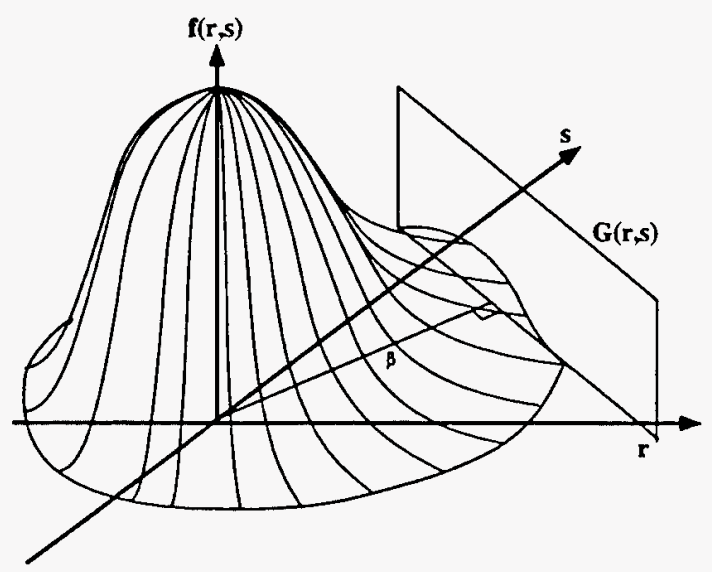

Figure 7. Depiction of joint probability density function and limit state function 


$$
\beta=\frac{-\nabla^{* T} \mathbf{u}^{*}}{\left(\nabla^{* T} \nabla\right)^{1 / 2}}=\frac{-\left.\sum_{i=1}^{n} u_{i}^{*}\left(\frac{\partial G}{\partial u_{i}}\right)\right|_{u=u^{*}}}{\sqrt{\sum_{i=1}^{n}\left(\frac{\partial G}{\partial u_{i}}\right)^{2}}}
$$

where the gradient is defined: $\nabla^{* T}=\left.\left(\frac{\partial G}{\partial u_{1}}, \frac{\partial G}{\partial u_{2}}, \cdots, \frac{\partial G}{\partial u_{n}}\right)\right|_{u=u^{*}}$

Ang and Tang (1984 p345) present an excellent first order interpretation of these results. As with the MV method, take a Taylor Series expansion of the system performance function, but this time expand about the MPP:

$$
G(\mathbf{x})=\left.G\left(x_{1}, \ldots, x_{n}\right) \approx G(\mathbf{x})\right|_{\mathbf{x}=\mathbf{x}^{*}}+\left.\sum_{i=1}^{n} \frac{\partial G(\mathbf{x})}{\partial x_{i}}\right|_{\mathbf{x}=\mathbf{x}^{*}}\left(x_{i}-x_{i}^{*}\right)+\text { H.O.T. }
$$

but since $\mathbf{x}^{*}$ is on the failure surface $\left.G(\mathbf{x})\right|_{\mathbf{x}=\mathbf{x}}{ }^{*}=0$. Putting the expression in terms of reduced variables:

$$
\begin{aligned}
& x_{i}-x_{i}^{*}=\left(\sigma_{i} u_{i}-\mu_{i}\right)-\left(\sigma_{i} u_{i}^{*}-\mu_{i}\right)=\sigma_{i}\left(u_{i}-u_{i}^{*}\right) \\
& \frac{\partial G(\mathbf{x})}{\partial x_{i}}=\frac{\partial G(\mathbf{u})}{\partial u_{i}}\left(\frac{\partial u_{i}}{\partial x_{i}}\right)=\frac{1}{\sigma_{i}}\left(\frac{\partial G(\mathbf{u})}{\partial u_{i}}\right)
\end{aligned}
$$

Therefore:

$$
\left.G(\mathbf{x}) \approx \sum_{i=1}^{n}\left(u_{i}-u_{i}^{*}\right) \frac{\partial G(\mathbf{u})}{\partial u_{i}}\right|_{u=\mathbf{u}^{*}}+\cdots
$$

Approximate expressions for the mean and variance of the system performance function can then be found (for independent random variables):

$$
\begin{aligned}
& \mu_{G} \approx-\left.\sum_{i=1}^{n} u_{i}^{*}\left(\frac{\partial G(\mathbf{u})}{\partial u_{i}}\right)\right|_{\mathbf{u}=\mathbf{u}^{*}} \\
& \left.\sigma_{G}^{2} \approx \sum_{i=1}^{n}\left(\frac{\partial G(\mathbf{u})}{\partial u_{i}}\right)^{2}\right|_{\mathbf{u}=\mathbf{u}^{*}}
\end{aligned}
$$

In terms of the approximate mean and variance, the safety index is:

$$
\beta=\frac{-\left.\sum_{i=1}^{n} u_{i}^{*}\left(\frac{\partial G(\mathbf{u})}{\partial u_{i}}\right)\right|_{\mathbf{u}=\mathbf{u}^{*}}}{\sqrt{\left.\sum_{i=1}^{n}\left(\frac{\partial G(\mathbf{u})}{\partial u_{i}}\right)^{2}\right|_{u=\mathbf{u}^{*}}}}=\frac{\mu_{G}}{\sigma_{G}}
$$

It should be noted that for the unique situation where the random variables are independent and the performance function is linear: $G(\mathbf{x})=a_{0}+\sum_{i=1}^{n} a_{i} x_{i}$ the expressions for performance moments 
$\mu_{G}, \sigma_{G}$, are exact. If, in addition, the random variables can be transformed into independent Gaussian random variables, then exact probabilistic statements can be made regarding the system performance: $p_{f}=\Phi\left(-\frac{\mu_{G}}{\sigma_{G}}\right)=\Phi(-\beta)$. Other than in these very limited situations, the probabilistic characterization of the system performance is only an approximation.

The set of reduced variables corresponding to the MPP can be found in a number of ways. It is obviously a straightforward nonlinear optimization problem. However, a number of algorithms have been suggested all generally following an approach involving the iterative solution of the equations:

$$
\begin{aligned}
\mathbf{u}_{i}^{*} & =-\gamma_{i} \beta \\
G\left(\mathbf{u}^{*}\right) & =0
\end{aligned}
$$

where:

$$
\gamma_{i}=\frac{\left.\left(\frac{\partial G(\mathbf{u})}{\partial u_{i}}\right)\right|_{\mathbf{u}=\mathbf{u}^{*}}}{\sqrt{\left.\sum_{i=1}^{n}\left(\frac{\partial G(\mathbf{u})}{\partial u_{i}}\right)^{2}\right|_{u=u^{*}}}}
$$

A typical approach:

1. Formulate the limit state in terms of the reduced variables.

2. Assume an initial value for $\mathbf{u}^{*}$. Typically the value for the first iteration is taken as the mean so $\mathbf{u}^{*}=(0,0, \ldots, 0)$

3. Evaluate the partial derivatives: $\left.\left(\frac{\partial G(\mathbf{u})}{\partial u_{i}}\right)\right|_{u=u^{\circ}}$

4. Evaluate each $\gamma_{i}$

5. Formulate each reduced variable in terms of the safety index: $u_{i}^{*}=-\gamma_{i} \beta$

6. Solve for $\beta$ given $G(\gamma \beta)=0$

7. Using the result from step 6 form a new candidate for MPP: $u_{i}^{*}=-\gamma_{i} \beta$

8. Repeat steps $2-7$ until convergence is reached

It should also be noted that the Hasofer-Lind approach is independent of the underlying distributions of the random variables. The estimation of the safety index can be accomplished independently of this information and even crude estimates of the failure probability can be made. However, if it is desired to accurately characterize performance in probabilistic terms, it is required that the distribution information be utilized to the greatest extent possible. 


\section{Application}

The application to be used is once again the stress voiding problem The original limit state function is repeated here for completeness:

$$
G\left(v_{0}, L\right)=A_{c}-v_{0}\left[0.01694-0.01353 \exp \left(-\frac{0.4158}{L}\right)\right]
$$

Since the random variables are independent, the first step is to transform the random variables into reduced space:

$$
\begin{gathered}
u_{1}=\frac{v_{0}-\mu_{v_{0}}}{\sigma_{v_{0}}} \rightarrow v_{0}=\mu_{v_{0}}+u_{1} \sigma_{v_{0}} \\
u_{2}=\frac{L-\mu_{L}}{\sigma_{L}} \rightarrow L=\mu_{L}+u_{2} \sigma_{L}
\end{gathered}
$$

The new limit function, now in terms of the reduced variables, is then given by the expression:

$$
G\left(\mu_{v_{0}}+u_{1} \sigma_{v_{0}}, \mu_{L}+u_{2} \sigma_{L}\right)=A_{c}-\left(\mu_{v_{0}}+u_{1} \sigma_{v_{0}}\right)\left[0.01694-0.01353 \exp \left(-\frac{0.4158}{\left(\mu_{L}+u_{2} \sigma_{L}\right)}\right)\right]
$$

\begin{tabular}{|c|c|c|c|c|}
\hline$u_{1}^{*}$ & $u_{2}^{*}$ & $\gamma_{1}$ & $\gamma_{2}$ & $\beta$ \\
\hline 2.0 & 2.0 & -0.9706 & 0.2407 & 1.1713 \\
\hline 1.1277 & -0.3195 & -0.9619 & 0.2733 & 1.1712 \\
\hline 1.1274 & -0.3204 & -0.9619 & 0.2733 & 1.1712 \\
\hline
\end{tabular}

Table 1. H-L Application Results 

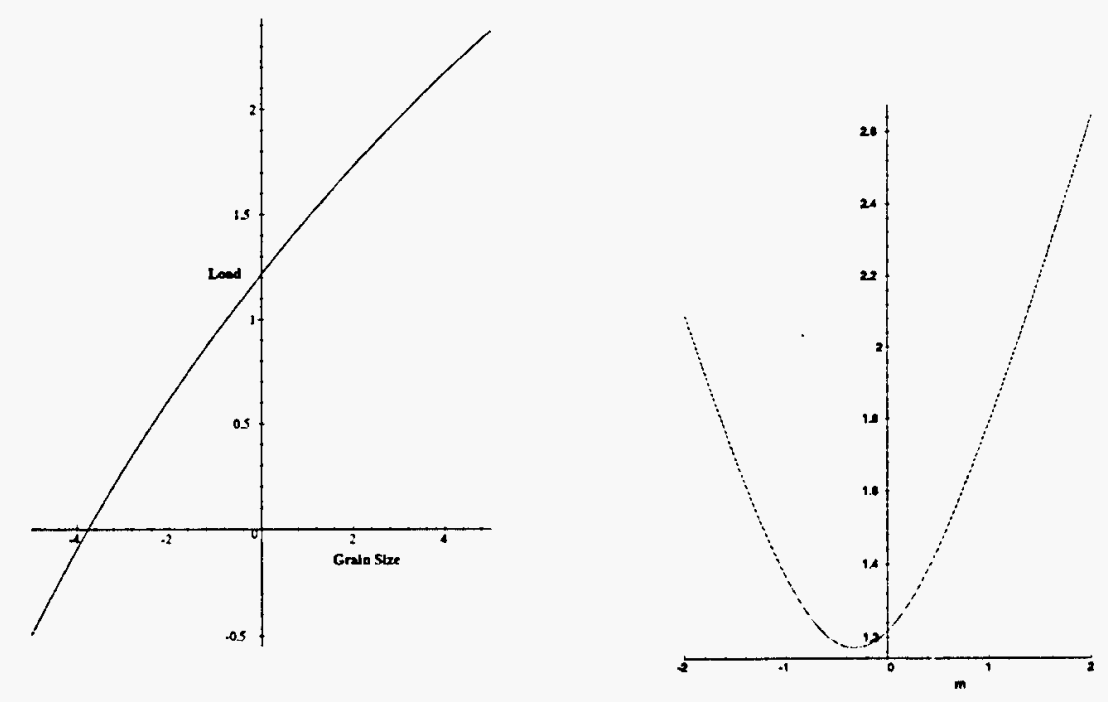

Figure 8. New Limit State Function and Distance Function

The new limit state function (in reduced space) is depicted in Figure 8. For a two dimensional problem such as this the minimum distance to the failure surface and the associated MPP can be found graphically. To demonstrate the algorithm a full set of iterations was performed. Table 1 . outlines the results from the intermediate steps.

\section{Intermediate Summary of Results}

The analytical techniques that follow begin to explicitly consider information regarding the underlying density function. Until this point, only information regarding the first and second moments of the density functions have been required. Before moving on to the next level techniques, it might be informative to contrast the results available thus far. Table 2 provides this summary. Note that for the RS method, only one formulation has been documented: the results from the second formulation (as in the MV method) would have been equally disastrous.

\begin{tabular}{|l|l|l|l|l|}
\cline { 2 - 5 } \multicolumn{1}{c|}{} & \multicolumn{1}{c|}{ Mean Value I } & Mean Value II & Response Surface & Hasofer-Lind \\
\hline$\mu_{G}$-mean & 0.5284 & 0.0018 & 0.5252 & 0.5370 \\
\hline$\sigma_{G}$-standard deviation & 0.4445 & 0.0508 & 0.4451 & 0.4514 \\
\hline
\end{tabular}

Table 2. Intermediate Results

(Please note that the similarity of the results of the MV and the $\mathrm{H}-\mathrm{L}$ methods is an artifact of the unfortunate choice of the problem chosen as an example. Future editions of this summary will contain additional examples.)

\section{Sensitivity Analysis}

Sensitivity analysis can provide information to support additional testing in an efficient manner. If the probabilistic characteristics of the response of a system are not significantly impacted by statistical variation in certain parameters, those parameters can (and possibly should) be omitted 
from the probabilistic analysis. In addition, testing efforts required to more fully characterize the density functions of important variables can be accomplished in a more concerted effort.

Sensitivity measures when used in the context of analytical methods are often referred to as importance factors. The magnitudes of these factors characterize the impact of each of the random variables on the safety index and thereby, their impact on the probability of failure.

The importance factors represent the direction cosines of the individual random variables in reduced space and are defined (see Figure 9):

$$
\gamma_{i}=\frac{-u_{i}^{*}}{\beta}=\frac{\left.\left(\frac{\partial G(\mathbf{u})}{\partial u_{i}}\right)\right|_{\mathbf{u}^{*} \mathbf{u}^{*}}}{\sqrt{\left.\sum_{i=1}^{n}\left(\frac{\partial G(\mathbf{u})}{\partial u_{i}}\right)^{2}\right|_{\mathbf{u}=\mathbf{u}^{*}}}}
$$

As a computation check it is noted that: $\sum_{i=1}^{n} \gamma_{i}^{2}=1$ and $-1 \leq \gamma_{i} \leq 1$.

These factors were implicitly discussed in the previous material and are calculated as a natural element in the analysis. In particular, refer to Table 1 . The importance factors for the initial stress, $v_{0}$, and the grain size, L, are: -0.9619 and 0.2733 , respectively. Clearly, the uncertainty in the initial stress is the dominant contributor to the probability that the system will fail.

\section{Rackwitz-Fiessler}

As previously mentioned, the above techniques do not rely on the availability of detailed probability density function information. As such these techniques are very commonly applied early in the analysis to get a feel for the importance of design variables. However, the next logical step in a complete probabilistic analysis is the incorporation of as much statistical information as possible. This was a key impetus for extending the Hasofer-Lind approach. Rackwitz and Fiessler (1978) suggested one approach improving the accuracy of $\Phi(-\beta)$ as an approximation to

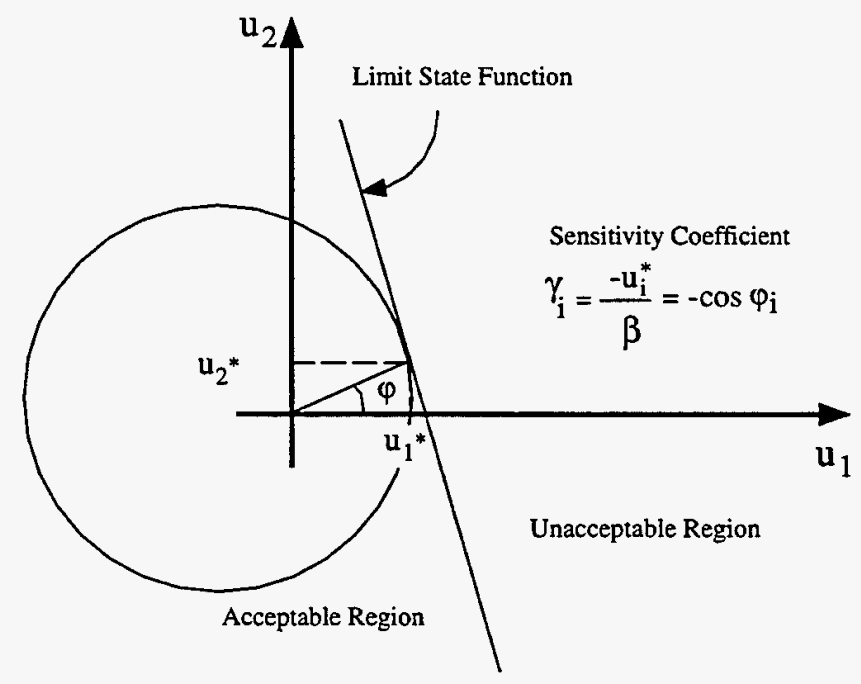

Figure 9. Depiction of Sensitivity Coefficients the true underlying probability density function of the system response.

When using $\Phi(-\beta)$ as an approximation, it is inherently assumed that the system response can be accurately represented (at least locally) by a linear combination of Gaussian distributed random variables. When the true underlying distributions are significantly non-Gaussian, the approximation can have significant error, particularly when investigating regions in the tails of the distributions. The Rackwitz-Fiessler (R-F) method, also referred to as the 'equivalent normal' (EN) method, is based on developing a better Gaussian approximation to the true probability density functions in the area of interest within the design space (i.e. $\mathbf{x}$-space). 


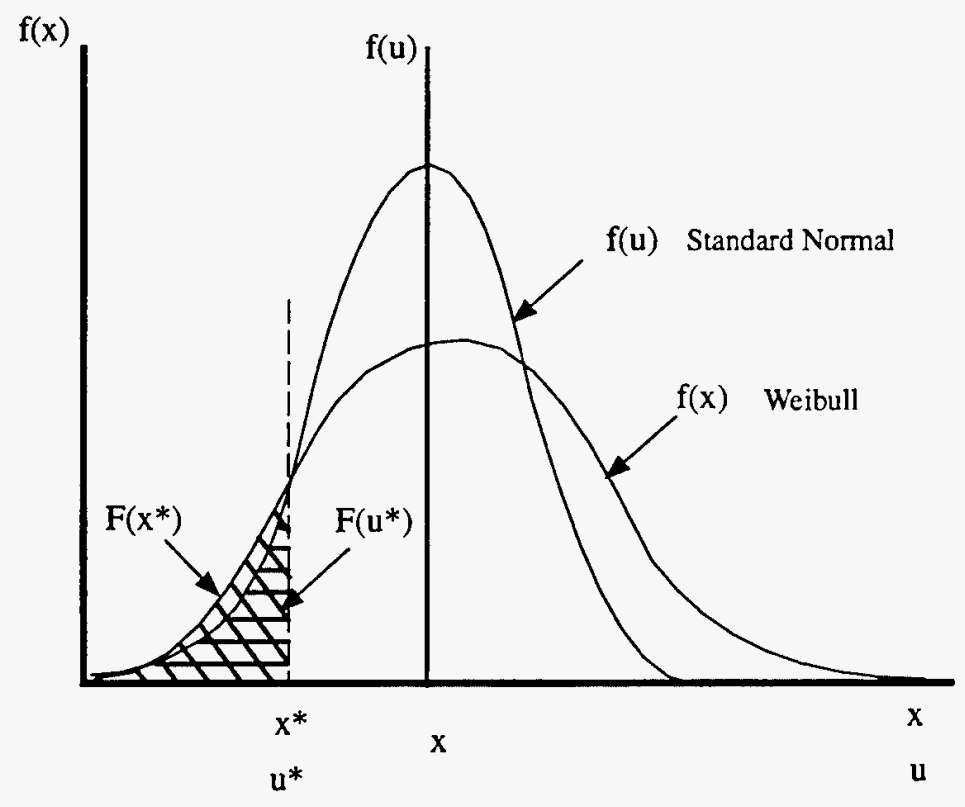

Figure 10. Gaussian Approximation

It is still assumed that the limit state function is approximately linear.

As mentioned, previous techniques have assumed that the true underlying probability density function associated with a design parameter can be approximated with a Gaussian distribution having the same first and second moments. Figure 10 depicts the approximation of Weibull probability density function with a Gaussian pdf. The Weibull density function has positive support while the Gaussian density function is defined over the entire real line. The accuracy is obviously very limited in the tail regions, and there is also no guarantee that the approximation is adequate even in the central regions of the probability density functions. The R-F technique forces the two density functions to have similar statistical properties in the area of primary interest in the probabilistic analysis; specifically in the region of the MPP.

Given a cumulative density function $F(x)$ and the associated density function $f(x)$. It is desired to find the mean $\mu^{\prime}$ and standard deviation $\sigma^{\prime}$ of an 'equivalent' Gaussian density function such that the cumulative density functions and the probability density functions at the MPP are both equivalent. That is:

$$
\begin{aligned}
& F\left(x^{*}\right)=\Phi\left(\frac{x^{*}-\mu^{\prime}}{\sigma^{\prime}}\right) \\
& f\left(x^{*}\right)=\frac{1}{\sigma^{\prime}} \phi\left(\frac{x^{*}-\mu^{\prime}}{\sigma^{\prime}}\right)
\end{aligned}
$$

where $\Phi(\cdot), \phi(\cdot)$ are the standard normal cdf and pdf respectively. Solving these two equations

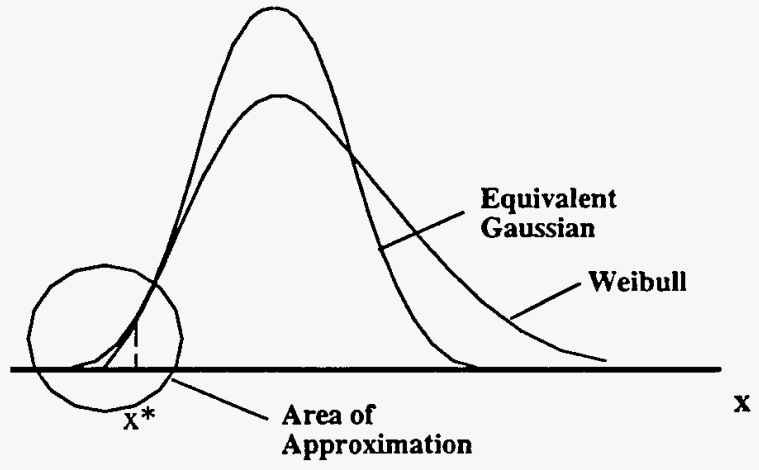

Figure 11. Equivalent Normal Approximation with two unknowns:

$$
\begin{aligned}
& \mu^{\prime}=x^{*}-\sigma^{\prime} \Phi^{-1}\left[F\left(x^{*}\right)\right] \\
& \sigma^{\prime}=\frac{\phi\left\{\Phi^{-1}\left[F\left(x^{*}\right)\right]\right\}}{f\left(x^{*}\right)}
\end{aligned}
$$

These two moments are then substituted into the moments used during the $\mathrm{H}-\mathrm{L}$ iterations. Note that since the equivalent normal parameters are functions of $\mathbf{x}^{*}$, the equivalent moments must be updated at the beginning of each H-L iteration. Figure 11 is a graphical depiction of the approximation. 


\section{Application}

Assume that the random variables, $\sigma_{0}$ and $\mathrm{L}$ are independent with known first and second moments, but now the exact form of the probability density functions are known. To permit comparisons, the moments of the distributions will remain the same. Let the initial stress on the interconnect be characterized by a Weibull distributed random variable $\sigma_{0} \sim W(300,0.2)$ with probability functions:

$$
\begin{aligned}
& f(x)=\frac{\alpha t^{\alpha-1}}{\eta^{\beta}} \exp \left[-\left(\frac{t}{\eta}\right)^{\alpha}\right], \quad t \geq 0 \\
& F(x)=1-\exp \left[-\left(\frac{t}{\eta}\right)^{\alpha}\right]
\end{aligned}
$$

Maintaining the previously defined moments the parameters are therefore: $\alpha=5.687$ and $\eta=324.34$. The grain size parameter will be assumed to be a lognormally distributed random variable $L \sim \operatorname{Ln}(1.25,0.1)$ :

$$
\begin{aligned}
& f(x)=\frac{1}{x \sqrt{2 \pi \xi}} \exp \left\{-\frac{[\ln (x)-\lambda]^{2}}{2 \xi^{2}}\right\} \\
& F(x)=\int_{-\infty}^{x} \frac{1}{s \sqrt{2 \pi \xi}} \exp \left\{-\frac{[\ln (s)-\lambda]^{2}}{2 \xi^{2}}\right\} d s
\end{aligned}
$$

The parameters of the distribution are therefore: $\lambda=0.2182$, and $\xi=0.09975$. Once again, the line width is assumed to be 3 microns and the critical crack length is assume to occur when it extends across $90 \%$ of the interconnect: $A_{c}=2.7$.

Since the random variables are independent, the first step is to transform the random variables into reduced space, now using the moments of the equivalent normal distribution:

$$
\begin{gathered}
u_{1}=\frac{\sigma_{0}-\mu_{\sigma_{0}}^{\prime}}{\sigma_{\sigma_{0}}^{\prime}} \rightarrow \sigma_{0}=\mu_{\sigma_{0}}^{\prime}+u_{1} \sigma_{\sigma_{0}}^{\prime} \\
u_{2}=\frac{L-\mu_{L}^{\prime}}{\sigma_{L}^{\prime}} \rightarrow L=\mu_{L}^{\prime}+u_{2} \sigma_{L}^{\prime}
\end{gathered}
$$

Using initial estimates of the equivalent normal moments, a full series of $\mathrm{H}-\mathrm{L}$ iterations is performed until the safety index converges. Using the new estimate of the MPP that results from the $\mathrm{H}-\mathrm{L}$ algorithm, a new set of moments for the equivalent normal distributions, a second application of $\mathrm{H}-\mathrm{L}$ is performed. This continues until once again the safety index converges. Table 3 outlines the results from the intermediate steps.

It should be noted that the quick convergence of both the H-L and R-F methods is common for performance functions without major discontinuities.

A major disadvantage of all the methods discussed thus far is the difficulty in addressing statistical dependency between the random variables. The last major development in analytical methods has been to improve the capability of analytical methods to explicitly include these dependencies. One approach to this problem is employed by the advanced first order reliability method. 


\begin{tabular}{|c|c|c|c|c|c|c|c|}
\hline$\mu_{1}^{\prime}$ & $\sigma_{1}^{\prime}$ & $\mu_{2}^{\prime}$ & $\sigma_{2}^{\prime}$ & $u_{1}^{*}$ & $u_{2}^{*}$ & $\beta$ & $\Phi(-\beta)$ \\
\hline 300 & 60 & 1.25 & 0.125 & 2.0 & 2.0 & 1.1713 & 0.1208 \\
\hline & & & & 1.1277 & -0.3195 & 1.1712 & 0.1208 \\
\hline 309.58 & 51.57 & 1.316 & 0.384 & 1.1274 & -0.3204 & 0.9777 & 0.1641 \\
\hline & & & & 0.6499 & -0.7303 & 0.9777 & 0.1641 \\
\hline 305.59 & 56.13 & 1.379 & 0.309 & 0.6472 & -0.7328 & \multicolumn{2}{|c}{} \\
\cline { 1 - 7 } & & &
\end{tabular}

Table 3. Results of Rackwitz-Fiessler Method

\section{Advanced First Order Reliability Method}

The advanced, first-order reliability method (AFORM) is one of the last major steps in addressing the difficulties with the previous techniques. This approach was proposed by Hohenbichler and Rackwitz in 1981 and will be referred to in this document either as AFORM or the H-R method. It includes the normalization procedure of the $\mathrm{H}-\mathrm{L}$ technique. In addition, it includes the probability density function approximation approach from the R-F method, and also addresses the issue of dependency among the random variables. Embedded within the iterative AFORM is a technique for transforming the original, non-Gaussian, dependent random variables into a set of independent, standardized Gaussian random variables. This latter feature is based on the general transformation method developed by Rosenblatt (1952). In the interests of completeness, the following discussion outlines the fundamental approach of the transformation method.

\section{Rosenblatt Transformation}

Notationally, $\mathbf{x}$ will refer to a vector of independent random variables, while $\mathbf{y}$ will be a vector of statistically dependent random variables. Let the joint probability and cumulative density functions of $\mathbf{y}=\left(y_{1}, y_{2}, \ldots, y_{n}\right)$ be defined as: $f(\mathbf{y})$ and $F_{Y}(\mathbf{y})$ respectively. Define the marginal density functions and cumulative marginal distribution functions:

$$
\begin{aligned}
f_{i}\left(y_{1}, y_{2}, \ldots, y_{i}\right) & =\int_{-\infty}^{\infty} \cdots \int_{-\infty}^{\infty} f_{i}\left(y_{1}, y_{2}, \ldots, y_{i}, s_{i+1}, \ldots, s_{n}\right) d s_{i+1} \ldots d s_{n} \\
H_{i}\left(y_{i} \mid y_{1}, y_{2}, \ldots, y_{i-1}\right) & =\frac{1}{K_{i}} \int_{-\infty}^{y_{i}} f_{i}\left(y_{1}, y_{2}, \ldots, y_{i-1}, s_{i}\right) d s_{i}
\end{aligned}
$$

where : $K_{i}=\int_{-\infty}^{\infty} f_{i}\left(y_{1}, y_{2}, \ldots, y_{i-1}, s_{i}\right) d s_{i}$ and $H_{1}\left(y_{1}\right)=F_{1}\left(y_{1}\right)$. The new set of independent, standardized Gaussian random variables are then given by:

$$
\mathbf{u}=\left(u_{1}, u_{2}, \ldots, u_{n}\right)=\left\{\Phi^{-1}\left[H_{1}\left(y_{1}\right)\right], \Phi^{-1}\left[H_{2}\left(y_{2} \mid y_{1}\right)\right], \ldots, \Phi^{-1}\left[H_{n}\left(y_{n} \mid y_{1}, y_{2}, \ldots, y_{n-1}\right)\right]\right\}
$$

Typically, the necessary conditional joint density functions are difficult to obtain except in rare situations. The alternative is to obtain the conditional density function through numerical means. It should be noted the Rosenblatt transformation reduces to the traditional rotation and translation transformation for dependent Gaussian distributed random variables.

A factor that certainly lends suspicion to the use of any transformation method is the very limited availability of data to support confident characterization of the joint density functions; it is common to resort to characterizing random variables in such a manner that analytical transformation techniques can be used. This difficulty exists regardless of whether the analysts are using 
analytical or Monte Carlo-based techniques. The point here is that gross assumptions about statistical dependency are often made with insufficient justification regardless of the uncertainty analysis method being used.

\section{Rosenblatt Transformation Examples}

This first example is due to Hohenbichler and Rackwitz (1981).Let the joint cumulative distribution function for two random variables $\mathrm{Y}_{1}, \mathrm{Y}_{2}$ be defined (for $y_{1}, y_{2} \geq 0$ ):

$$
F\left(y_{1}, y_{2}\right)=1-\exp \left(-y_{1}\right)-\exp \left(-y_{2}\right)+\exp \left(-y_{1}-y_{2}-\theta y_{1} y_{2}\right)
$$

Therefore:

$$
\begin{array}{ll}
\Phi\left(u_{1}\right)=H_{1}\left(y_{1}\right) & \Rightarrow u_{1}=\Phi^{-1}\left[H_{1}\left(y_{1}\right)\right] \\
\Phi\left(u_{2}\right)=H_{2}\left(y_{2} \mid y_{1}\right) & \Rightarrow u_{2}=\Phi^{-1}\left[H_{2}\left(y_{2} \mid y_{1}\right)\right]
\end{array}
$$

where:

$$
\begin{aligned}
H_{1}\left(y_{1}\right) & =1-\exp \left(-y_{1}\right) \\
H_{2}\left(y_{2} \mid y_{1}\right) & =\frac{\int_{0}^{x_{2}} f\left(y_{1}, s\right) d s}{f\left(y_{1}\right)}=\frac{\exp \left(-y_{1}\right)-\left(1+\theta y_{2}\right) \exp \left(-y_{2}-\theta y_{1} y_{2}\right)}{\exp \left(-y_{1}\right)}
\end{aligned}
$$

and finally:

$$
\mathbf{u}=\left\{\Phi^{-1}\left[1-\exp \left(-y_{1}\right)\right], \quad \Phi^{-1}\left[1-\left(1+\theta y_{2}\right) \exp \left(-y_{2}-\theta y_{1} y_{2}\right)\right]\right\}^{T}
$$

Notationally, we will denote this vector of standardized, independent random variables as:

$\mathbf{u}^{*}=\left\{u_{1}^{*}, \ldots, u_{n}^{*}\right\}=\left.\mathbf{u}\right|_{\mathbf{y}^{*}}$, where the subscript indicates evaluation of the transformation at a particular point, $\mathbf{y}^{*}$.

This second example is from Ang and Tang (1984). Let $Y_{1}$ be a Gaussian distributed random variable with mean $\mu_{1}$ and standard deviation $\sigma_{1}$. Let $Y_{2}$ be a lognormally distributed random variable with probability density function:

$$
f(y)=\frac{1}{y \xi \sqrt{2 \pi}} \exp \left[-\frac{1}{2}\left\{\frac{\ln (y)-\lambda}{\xi}\right\}\right] .
$$

Assume that $\mathrm{Y}_{1}, \mathrm{Y}_{2}$ are correlated such that $\rho_{12}=0.6$. Applying the Rosenblatt Transformation:

where:

$$
\begin{aligned}
& \Phi\left(u_{1}\right)=H_{1}\left(y_{1}\right) \\
& \Phi\left(u_{2}\right)=H_{2}\left(y_{2} \mid y_{1}\right)
\end{aligned}
$$

$$
H_{2}\left(y_{2} \mid y_{1}\right)=\Phi\left[\frac{\ln \left(y_{2}\right)-\lambda-\eta\left(\xi / \sigma_{1}\right)\left(y_{1}-\mu_{1}\right)}{\xi \sqrt{1-\eta^{2}}}\right]
$$

and:

$$
\eta=\frac{\rho}{\xi}\left(\frac{\sigma_{2}}{\mu_{2}}\right)
$$


The new vector of reduced space random variables is therefore composed of:

$$
\begin{aligned}
& u_{1}=\frac{y_{1}-\mu_{1}}{\sigma_{1}} \\
& u_{2}=\frac{\ln \left(y_{2}\right)-\lambda-\eta\left(\xi / \sigma_{1}\right)\left(y_{1}-\mu_{1}\right)}{\xi \sqrt{1-\eta^{2}}}
\end{aligned}
$$

Note that since the lognormal distribution function can be transformed into a Gaussian distribution function, the reduced space random variables could have been characterized without resorting to the Rosenblatt Transformation (and the results are identical).

\section{AFORM Algorithm}

The Advanced First Order Reliability Method can be summarized in a rather straightforward series of operations. As with the previously discussed methods, a point in design space is chosen to initiate the algorithm. While not necessarily the best place to start, the most common point is the vector of nominal or mean values $\mathbf{y}^{*}=\left\{\mu_{1}, \mu_{2}, \ldots, \mu_{n}\right\}$.

1. Using the Rosenblatt transformation, find the vector of standardized, independent random variables evaluated at the current design point: $\mathbf{u}^{*}=\left.\mathbf{u}\right|_{y}$.

2. Calculate the Jacobian transformation matrix evaluated at the current design point:

$$
\mathbf{J}=\left[\left.\begin{array}{cccc}
\frac{\partial u_{1}}{\partial y_{1}} & \frac{\partial u_{1}}{\partial y_{2}} & \cdots & \frac{\partial u_{1}}{\partial y_{n}} \\
\frac{\partial u_{2}}{\partial y_{1}} & \frac{\partial u_{2}}{\partial y_{2}} & & \vdots \\
\vdots & & \ddots & \\
\frac{\partial u_{n}}{\partial y_{1}} & \cdots & & \frac{\partial u_{n}}{\partial y_{n}}
\end{array}\right|_{y} .\right.
$$

For convenience note that:

$$
\frac{\partial u_{i}}{\partial y_{j}}=\frac{\partial \Phi^{-1}\left[H\left(y_{i} \mid y_{1}, \ldots\right)\right]}{\partial y_{j}}=\frac{1}{\phi\left(u_{i}\right)} \frac{\partial H\left(y_{i} \mid y_{1}, \ldots\right)}{\partial y_{j}}
$$

3. Evaluate the performance function and the associated gradient at the current design point:

$$
\begin{aligned}
g\left(\mathbf{y}^{*}\right) & =g\left(\mathbf{u}^{*}\right) \\
\nabla^{*} & =\nabla_{g\left(\mathbf{u}^{*}\right)}=\left(\mathbf{J}^{-1}\right)^{T} \nabla_{g\left(\mathbf{y}^{*}\right)}
\end{aligned}
$$

where the gradient is defined:

$$
\nabla_{g\left(\mathbf{u}^{\circ}\right)}=\left.\left\{\frac{\partial g}{\partial u_{1}}, \frac{\partial g}{\partial u_{2}}, \ldots \frac{\partial g}{\partial u_{n}}\right\}\right|_{u^{*}}
$$

4. Move to a new design point and calculate a new safety index:

$$
\begin{array}{ll}
\tilde{\mathbf{u}}^{*}=\frac{1}{\left(\nabla^{*}\right)^{T} \nabla^{*}}\left[\left(\nabla^{*}\right)^{T} \mathbf{u}^{*}-g\left(\mathbf{u}^{*}\right)\right] \nabla^{*} & \text { (reduced space) } \\
\tilde{y}^{*} \approx y^{*}+\mathbf{J}^{-1}\left(\tilde{\mathbf{u}}^{*}-\mathbf{u}^{*}\right) & \text { (design space - approximate) }
\end{array}
$$


and the safety index is then:

$$
\begin{aligned}
& |\beta|=\left(\mathbf{u}^{* T} \mathbf{u}^{*}\right)^{1 / 2} \text { or } \\
& \beta=\left[\frac{-\nabla^{*}}{\left|\nabla^{*}\right|}\right]^{T} \tilde{\mathbf{u}}^{*}
\end{aligned}
$$

5. Using the results from Step 4 as the new expansion point, repeat steps $1-4$ until convergence is reached for the safety index

\section{Application}

Assume that the initial stress $\sigma_{0}$ is a Gaussian distributed random variable with mean $\mu_{1}=300$ and coefficient of variation: $\kappa_{1}=0.2$. Let the grain size $L$ be a lognormally distributed random variable with median and coefficient of variation: $\tilde{y}_{2}=1.25, \kappa_{2}=0.1$. The initial stress and grain size will be assumed to be negatively correlated with correlation coefficient: $\rho=-0.5$. As before, the line width is 3 microns and the critical crack length is $90 \%$ of the interconnect width: $A_{c}=2.7$.

Applying the Rosenblatt Transformation:

$$
\begin{aligned}
& u_{1}=\Phi^{-1}\left[H\left(y_{1}\right)\right]=\frac{y_{1}-\mu_{1}}{\sigma_{1}} \\
& u_{2}=\frac{\ln \left(y_{2}\right)-\lambda-\eta\left(\xi / \sigma_{1}\right)\left(y_{1}-\mu_{1}\right)}{\xi \sqrt{1-\eta^{2}}}
\end{aligned}
$$

Using some useful relationships for the lognormal probability density function:

$$
\begin{aligned}
\lambda & =\ln \left(\tilde{y}_{2}\right)=\ln \left(\mu_{2}\right)-\frac{1}{2} \ln \left(1+\kappa_{2}^{2}\right)=\ln (1.25)=0.2231 \\
\mu_{2} & =\tilde{y}_{2} \sqrt{1+\kappa_{2}^{2}}=1.2563 \\
\xi & =\sqrt{\ln \left(1+\kappa_{2}^{2}\right)}=0.09975 \\
\sigma_{2} & =\kappa_{2} \mu_{2}=0.12563
\end{aligned}
$$

and:

$$
\eta=\frac{\rho}{\xi}\left(\frac{\sigma_{2}}{\mu_{2}}\right)=\frac{-0.5}{0.09975}(0.1)=-0.50125
$$

Therefore:

$$
\begin{aligned}
u_{1} & =\frac{y_{1}-\mu_{1}}{\sigma_{1}}=\frac{y_{1}-300}{60} \\
u_{2} & =\frac{\ln \left(y_{2}\right)-\lambda-\eta\left(\xi / \sigma_{1}\right)\left(y_{1}-\mu_{1}\right)}{\xi \sqrt{1-\eta^{2}}} \\
& =\frac{\ln \left(y_{2}\right)-0.2231+0.50125(0.09975 / 60)\left(y_{1}-300\right)}{0.09975 \sqrt{1-(-0.50125)^{2}}} \\
& =y_{1} 4.61+11.59 \ln \left(y_{2}\right)-1385.8
\end{aligned}
$$


The Jacobian can then be determined and evaluated at the current design point, $\mathrm{y}^{*}=\left\{\mu_{1}, \mu_{2}\right\}=\{300,1.2563\}$ :

$$
\mathbf{J}=\left[\begin{array}{cc}
\frac{1}{\sigma_{1}} & 0 \\
\frac{-\eta}{\sigma_{1} \sqrt{1-\eta^{2}}} & \frac{1}{\mathrm{y}_{2} \xi \sqrt{1-\eta^{2}}}
\end{array}\right]=\left[\begin{array}{cc}
0.1667 & 0 \\
0.00965 & 9.222
\end{array}\right]
$$

Given the original performance function:

$$
\begin{aligned}
& \mathrm{g}\left(\mathrm{y}_{1}, \mathrm{y}_{2}\right)=2.7-\mathrm{y}_{1}\left[0.01694-0.01353 \exp \left(-\frac{0.4158}{\mathrm{y}_{2}}\right)\right] \\
& \mathrm{g}(300,1.2563)=0.5333
\end{aligned}
$$

The gradients can also be calculated and evaluated:

$$
\begin{aligned}
\nabla_{g\left(y^{*}\right)} & =\left.\left\{\frac{\partial g}{\partial y_{1}}, \frac{\partial g}{\partial y_{2}}\right\}^{\mathrm{T}}\right|_{y^{*}} \\
& =\left.\left\{-0.01694-0.01353 \exp \left[-\frac{0.41589}{y_{2}}\right], 0.00562 \frac{y_{1}}{y_{2}^{2}} \exp \left[-\frac{0.41589}{y_{2}}\right]\right\}^{T}\right|_{y^{*}} \\
& =\{-0.00722,0.7680\}^{T} \\
\nabla^{*} & =\left(J^{-1}\right)^{T} \nabla_{g\left(y^{*}\right)} \\
& =\left[\begin{array}{cc}
60 & -0.0628 \\
0 & 0.1084
\end{array}\right]^{T}\left\{\begin{array}{c}
-0.00722 \\
0.7680
\end{array}\right\} \\
& =\{-0.4816,0.0833\}^{T}
\end{aligned}
$$

The new design point (in reduced space) and the associated safety index are therefore:

$$
\begin{aligned}
& \tilde{\mathbf{u}}^{*}=\frac{1}{\left(\nabla^{*}\right)^{T} \nabla^{*}}\left[\left(\nabla^{*}\right)^{T} \mathbf{u}^{*}-g\left(\mathbf{u}^{*}\right)\right] \nabla^{*}
\end{aligned}
$$

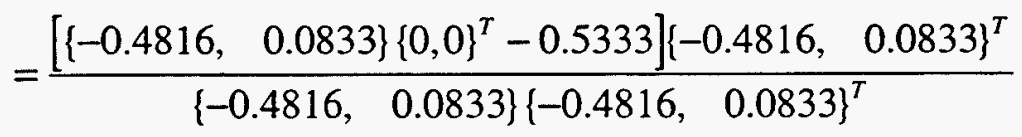

$$
\begin{aligned}
& =\{-1.0752,0.1859\}^{T} \\
& \beta=\left(\mathbf{u}^{* T} \mathbf{u}^{*}\right)^{1 / 2}=1.091
\end{aligned}
$$


and in the original design space:

$$
\begin{aligned}
\tilde{\mathbf{y}}^{*} & \approx \mathbf{y}^{*}+\mathbf{J}^{-1}\left(\mathbf{u}^{*}-\tilde{\mathbf{u}}^{*}\right) \\
& =\{300,1.2563\}^{T}+\left[\begin{array}{cc}
60 & 0 \\
-0.0628 & 0.1084
\end{array}\right]\left[\left\{\begin{array}{l}
0 \\
0
\end{array}\right\}-\left\{\begin{array}{c}
-1.0752 \\
0.1859
\end{array}\right\}\right] \\
& =\{364.5,1.168\}^{T}
\end{aligned}
$$

These results are then used as input to the next iteration. Table 4 summarizes the input and results from five iterations of the algorithm.

\begin{tabular}{|c||c|c|c||c|c|c|c||}
\multicolumn{1}{c||}{} & \multicolumn{5}{c||}{ Input } & \multicolumn{5}{c||}{ Result } \\
\hline Iteration & $\mathbf{y}^{*}$ & $\mathbf{x}^{*}$ & $\mathrm{~g}\left(\mathbf{x}^{*}\right)$ & $\mu_{\mathrm{g}}^{*}$ & $\sigma_{\mathrm{g}}^{*}$ & $\beta$ & $\Phi(-\beta)$ \\
\hline 1 & $\{300,1.2563\}$ & $\{0,0\}$ & 0.5333 & $\begin{array}{c}0.533 \\
3\end{array}$ & 0.4887 & 1.0911 & 0.1376 \\
\hline 2 & $\{364.5,1.1686\}$ & $\{1.075,-0.698\}$ & -0.0195 & 0.602 & 0.5201 & 1.1574 & 0.1235 \\
\hline 3 & $\{367.98,1.212\}$ & $\{1.133,-0.354\}$ & -0.0009 & $\begin{array}{l}0.603 \\
8\end{array}$ & 0.5118 & 1.1797 & 0.1190 \\
\hline 4 & $\{369.3,1.222\}$ & $\{1.155,-0.2412\}$ & -0.00004 & $\begin{array}{l}0.604 \\
6\end{array}$ & 0.5100 & 1.186 & 0.1179 \\
\hline 5 & $\{369.6,1.225\}$ & $\{1.161,-0.2427\}$ & $-0.2 \mathrm{e}-5$ & 0.604 & 0.5095 & 1.187 & 0.1176 \\
\hline
\end{tabular}

Table 4. Summary of Hohebichler-Rackwitz Iterations

\section{Summary}

The purpose of this report was to document and, when appropriate, identify strengths and weaknesses of an initial family of analytical techniques. As noted earlier, discussion was directed at the engineer or analyst whose background may involve an undergraduate probability course. The report may therefore appear to be simplistic to those with a much stronger probability and statistics background. It is important to note that the overall goal of this report is to assist the design engineer in moving away from the use of safety factors as the sole criteria for component design. As material and manufacturing costs escalate, alternative design methodologies must be explored. In addition, as design criteria move away from the component to system level specifications, the use of safety factors or margins of safety becomes difficult and generally overly conservative. With proper application, probabilistic methods have been shown to result in designs with $10-35 \%$ reduction in materials in conjunction with a reduced likelihood of failure, when compared to similar safety factor based designs.

However, while the goal is to encourage the use of these analytical methods by design engineers, there is a place for these methods in the field of risk analysis as well as the much broader area of uncertainty analysis. The methods outlined in this report, some almost 50 years old, are generally unknown in the field of uncertainty analysis. And, while uncertainty analysis has flourished without these techniques, there is room for new ideas and concepts. Hopefully this report will 
provide an introduction to the growing area of probabilistic methods for those statisticians and analysts involved with risk, reliability or uncertainty analysis.

The second part of this report entitled: A Survey of Probabilistic Methods Used in Reliability and Uncertainty Analysis: Analytical Methods II, will build on the analysis techniques

discussed in this first segment. While the majority of the techniques developed since 1985 have been variations of the methods outlined here, new and exciting approaches have been developed that will also be discussed.

\section{References}

Ang, A., W. H. Tang, 1984, "Probability Concepts in Engineering Planning and Design -Volume II: Decision, Risk and Reliability," Wiley and Sons, New York.

Freudenthal, A. M., 1956, "Safety and the Probability of Structural Failure," Transactions of the ASCE, Vol. 121, 1337-1397.

Hasofer, A., N. Lind, 1974, "An Exact and Invariant First-order Reliability Format," Journal of the Engineering Mechanics Division, ASCE, Vol. 100, No. EM1, pp111-121.

Hohenbichler, M., R. Rackwitz, 1981, "Non-Normal Dependent Vectors in Structural Safety," Journal of the Engineering Mechanics Division, ASCE, Vol. 107, No. EM6, December, pp1227-1238.

Iman, R. L., J. C. Helton, 1985, “A Comparison of Uncertainty and Sensitivity Analysis for Computer Models," NUREG/CR-3904, SAND84-1461, Sandia National Laboratories, Albuquerque, New Mexico.

Kapur, K., L. Lamberson, 1977, "Reliability in Engineering Design," Wiley and Sons, New York.

Morgan, M. G., M. Henrion, 1990, "Uncertainty: A Guide to Dealing with Uncertainty in Quantitative Risk and Policy Analysis," Cambridge University Press, New York.

Morris, M. D., 1991, "Factorial Sampling Plans for Preliminary Computational Experiments," Technometrics, Vol. 332, No. 2, pp161-174.

Rackwitz, R., B. Fiessler, 1978, "Structural Reliability Under Combined Random Load Sequences," Computers and Structures, Vol. 9, pp489-494.

Rosenblatt, M., 1952, "Remarks on a Multivariate Transformation," Annals of Mathematical Statistics, Vol. 23, No. 3, September, pp470-472.

Shinozuka, M., 1983, "Basis Analysis of Structural Safety,", Journal of Structural Division, ASCE, Vol. 3, No. 109, March.

Zimmerman, D. A., K.K. Wahl, A. L. Gutjahr, P. A. Davis, 1989, “A Review of Techniques for Propagating Data and Parameter Uncertainties in High-Level Radioactive Waste Repository Performance Assessment Models," NUREG/CR-5393, SAND89-1432, Sandia National Laboratories, Albuquerque, New Mexico. 


\section{Distribution}

$\begin{array}{llll}1 & \text { MS 1407 } & (1805) & \text { R. J. Salzbrenner } \\ 1 & \text { MS 0340 } & (1832) & \text { W. R. Cieslak } \\ 1 & \text { MS 0340 } & (1832) & \text { J. W. Braithwaite } \\ 1 & \text { MS 0340 } & (1832) & \text { N. R. Sorensen } \\ 1 & \text { MS 1411 } & (1833) & \text { F. M. Hosking } \\ 1 & \text { MS 1411 } & (1833) & \text { P. T. Vianco } \\ 1 & \text { MS 1411 } & (1834) & \text { F. G. Yost } \\ 1 & \text { MS 0708 } & (6214) & \text { P. S. Veers } \\ 1 & \text { MS 0455 } & (6232) & \text { L. R. Gilliom } \\ 1 & \text { MS 0746 } & (6411) & \text { R. M. Cranwell } \\ 1 & \text { MS 0746 } & (6411) & \text { D. J. Anderson } \\ 1 & \text { MS 0746 } & (6411) & \text { L. A. Painton-Swiler } \\ 20 & \text { MS 0746 } & (6411) & \text { D. G. Robinson } \\ 1 & \text { MS 0747 } & (6412) & \text { G. D. Wyss } \\ 1 & \text { MS 0405 } & (6413) & \text { A. S. Benjamin } \\ 1 & \text { MS 9102 } & (8402) & \text { M. W. Perra } \\ 1 & \text { MS 0177 } & (9003) & \text { P. D. Van Buren } \\ 1 & \text { MS 0841 } & (9100) & \text { P. M. Hommert } \\ 1 & \text { MS 0835 } & (9113) & \text { B. F. Blackwell } \\ 1 & \text { MS 0835 } & (9113) & \text { V. J. Romero } \\ 1 & \text { MS 0819 } & (9231) & \text { A. C. Robinson } \\ 1 & \text { MS 0819 } & (9231) & \text { T. G. Trucano } \\ 1 & \text { MS 0439 } & (9234) & \text { D. R. Martinez } \\ 1 & \text { MS 0439 } & (9234) & \text { J. R. Red-Horse } \\ 1 & \text { MS 0439 } & (9234) & \text { M. S. Eldred } \\ 1 & \text { MS 0557 } & (9741) & \text { T. L. Paez } \\ 1 & \text { MS 0829 } & (12323) & \text { K. V. Diegert } \\ 1 & \text { MS 0829 } & (12323) & \text { B. M. Rutherford } \\ 1 & \text { MS 0405 } & (12333) & \text { M. P. Bohn } \\ 1 & \text { MS 9018 } & (8940-2) & \text { Central Technical Files } \\ 5 & \text { MS 0899 } & (4916) & \text { Technical Library } \\ 2 & \text { MS 0619 } & (12690) & \text { Review \& Approval Desk for } \\ & & & \text { DOE/OSTI } \\ & & & \end{array}$

TOTAL COPIES $=46$ 

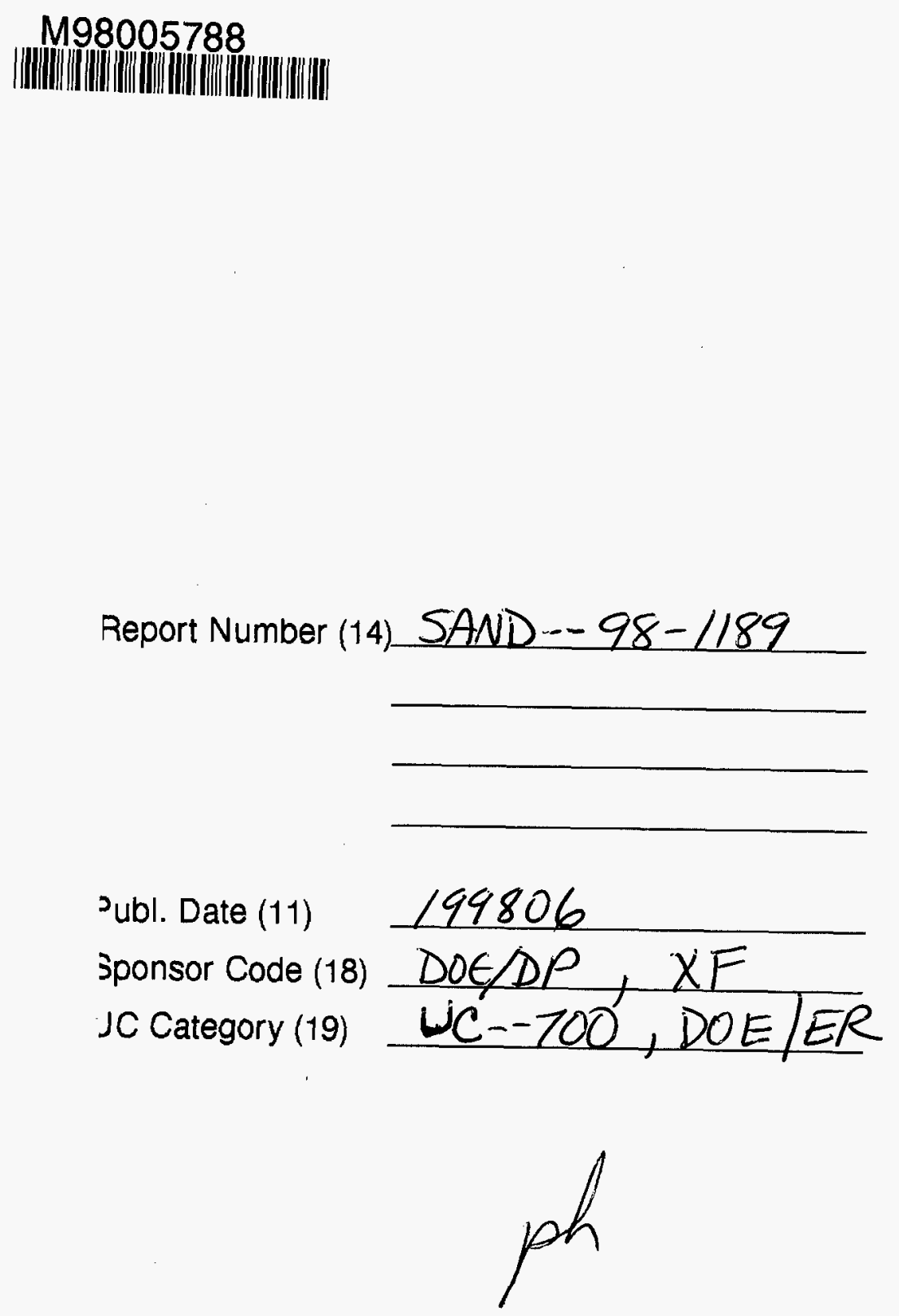

19980720030

DTC QUALTTY RTOFAGPQ $\mathbf{5}$ 